\title{
Higher Order Perturbation Theory of Exponential Lagrangians: Fourth Order *
}

\author{
K. Pohlmeyer
}

Instituto de Fisica, Universidade Federal do Rio Grande do Sul, Pôrto Alegre RS, Brazil and

II. Institut für Theoretische Physik der Universität Hamburg, Federal Republic of Germany

Received October 5, 1973

\begin{abstract}
We define the vacuum expectation value of the time-ordered product of four exponentials of free massless scalar fields as a continuous linear functional over a suitable test function space using minimal singularity as a criterion.
\end{abstract}

\section{Introduction}

The structure of the second and third order terms in a perturbation theoretic expansion of the Green's functions in powers of the exponential interaction Lagrangian $G \cdot L_{\text {int }}(x)=G: \exp (f \phi(x))-1:, \phi(x)$ being a free massless scalar field, has been analyzed by several authors [1-4].

The position taken in Ref. [1] and [2] can be described as follows: The problem of defining the time-ordered products $T\left(x_{1}, \ldots, x_{n}\right)$ $=i^{n+1} T L_{\text {int }}\left(x_{1}\right) \ldots L_{\text {int }}\left(x_{n}\right)$ is equivalent to the problem of defining the connected parts of the vacuum expectation values $\tau\left(x_{1}, \ldots, x_{n}\right)$ of the time-ordered products. In fact, owing to the formula

$$
\begin{gathered}
T: \exp \left(f \phi\left(x_{1}\right)\right): \ldots: \exp \left(f \phi\left(x_{n}\right)\right): \\
=\left\{\prod_{1 \leqq j<k \leqq n} \exp \left[-\lambda /\left(\left(x_{j}-x_{k}\right)^{2}-i O\right)\right]\right\}: \exp \left(f \phi\left(x_{1}\right)\right) \ldots \exp \left(f \phi\left(x_{n}\right)\right):,
\end{gathered}
$$

where $\lambda$ stands for $f^{2} / 4 \pi^{2}$, the combinatorics is particularly simple. In a successive construction of the time-ordered vacuum expectation values along Bogoliubov's lines [5], $\tau\left(x_{1}, \ldots, x_{n}\right)$ is determined by $\tau\left(x_{1}, \ldots, x_{r}\right)$ with $r<n$ via locality and unitarity only up to an arbitrary real, Lorentz invariant, localizable [6], symmetric distribution with support in the points where all $n$ arguments $x_{1}, \ldots, x_{n}$ coincide. The removal of this arbitrariness is our main concern.

Assuming that all $\tau\left(x_{1}, \ldots, x_{r}\right)$ with $r<n$ have already been constructed, we confine our attention to the real part $\mathscr{R} e \tau\left(x_{1}, \ldots, x_{n}\right)$ since

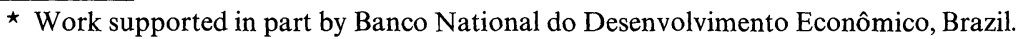


this is the only undetermined part. Moreover, since time-ordering involves multiplication of well-defined objects such as the vacuum expectation values of the products of $n$ interaction Lagrangians in various orders by step functions that depend on the time (-difference) variables only, we average $\mathscr{R} e \tau\left(x_{1}, \ldots, x_{n}\right)$ over the spatial variables with sufficiently smooth real test functions $f$ and study the resulting distributions $\mathscr{R} e \tau_{f}$ in the time variables near the points where all $n$ arguments $x_{1}^{0}, \ldots, x_{n}^{0}$ coincide.

In [1] it has been shown that for any admissable choice of $\tau\left(x_{1}, x_{2}\right)$ the contribution to $\mathscr{R} e \tau_{f}\left(x_{1}^{0}, x_{2}^{0}\right)$ from the points $x_{1}^{0}=x_{2}^{0}$ can be separated from the rest. By requiring the absence of a singular contribution from these coinciding times we arrive at a uniquely determined, least singular definition for $\mathscr{R} e \tau_{f}\left(x_{1}^{0}, x_{2}^{0}\right)$ and, moreover, these individual definitions (for every real, sufficiently smooth spatial test function $f$ ) can be derived from one particular Lorentz invariant definition of $\mathscr{R} e \tau\left(x_{1}, x_{2}\right)$. Thus we are led to a least singular choice for $\mathscr{R} e \tau\left(x_{1}, x_{2}\right)$ and thereby to a least singular definition of $\tau\left(x_{1}, x_{2}\right)$, the superpropagator $E_{F}\left(x_{1}-x_{2}\right)$.

In [2] we took this definition of $\tau\left(x_{1}, x_{2}\right)$ and showed that for any admissable choice of $\tau\left(x_{1}, x_{2}, x_{3}\right)$, as before, the contribution to $\mathscr{R} e \tau_{f}\left(x_{1}^{0}, x_{2}^{0}, x_{3}^{0}\right)$ from the points $x_{1}^{0}=x_{2}^{0}=x_{3}^{0}$ can be separated from the rest. Again, by requiring the absence of a singular contribution from these coinciding times we arrive at a uniquely determined least singular definition of $\mathscr{R} e \tau_{f}\left(x_{1}^{0}, x_{2}^{0}, x_{3}^{0}\right)$. We note that these individual definitions for every real, sufficiently smooth spatial test function $f$ are just the corresponding spatial averages of one particular Lorentz invariant choice for $\mathscr{R} e \tau\left(x_{1}, x_{2}, x_{3}\right)$. This least singular definition of $\mathscr{R} e \tau\left(x_{1}, x_{2}, x_{3}\right)$ is uniquely determined. We were able to give the least singular timeordered vacuum expectation value $\tau\left(x_{1}, x_{2}, x_{3}\right)$ in an explicit form.

In the present paper we go one step beyond the results of Ref. [2] by considering the definition problem for $\tau\left(x_{1}, \ldots, x_{4}\right)$, the time-ordered vacuum expectation value of four interaction Lagrangians or rather its connected part, taking the least singular definitions of $\tau\left(x_{1}, x_{2}\right)$ and $\tau\left(x_{1}, x_{2}, x_{3}\right)$. Here, for the first time in our approach we encounter a situation that corresponds to the occurence of overlapping divergencies in the perturbation theoretic treatment of renormalizable Lagrangian field theories. It is therefore interesting to find out whether the criterion of minimal singularity is still meaningful and whether it can be used to eliminate completely the arbitrariness in defining this time-ordered vacuum expectation value.

To this end, we shall analyze the structure of $\tau\left(x_{1}, \ldots, x_{4}\right)$ or rather of $\mathscr{R e} \tau_{f}\left(x_{1}^{0}, \ldots, x_{4}^{0}\right)$ outside, but close to points whose time components totally coincide. 
If $\mathfrak{\Im}_{4}$ denotes the permutation group of four objects and if its elements $\sigma$ are represented by

$$
\sigma=\left(\begin{array}{llll}
1 & 2 & 3 & 4 \\
i & j & k & l
\end{array}\right)
$$

we may formally decompose $\tau\left(x_{1}, \ldots, x_{4}\right)$ as follows

$$
\begin{aligned}
\tau\left(x_{1}, \ldots, x_{4}\right)=\frac{i}{2} \sum_{\sigma \in \mathscr{E}_{4}}\left[i E_{F}\left(x_{i}-x_{j}\right)\right]\left[i E_{F}\left(x_{j}-x_{k}\right)\right]\left[i E_{F}\left(x_{k}-x_{l}\right)\right] \\
\quad+\frac{i}{6} \sum_{\sigma \in \mathscr{E}_{4}}\left[i E_{F}\left(x_{i}-x_{j}\right)\right]\left[i E_{F}\left(x_{i}-x_{k}\right)\right]\left[i E_{F}\left(x_{i}-x_{l}\right)\right] \\
\quad+\frac{i}{2} \sum_{\sigma \in \mathfrak{E}_{4}}\left[i E_{F}\left(x_{i}-x_{j}\right)\right]\left[i E_{F}\left(x_{j}-x_{k}\right)\right]\left[i E_{F}\left(x_{k}-x_{i}\right)\right]\left[i E_{F}\left(x_{i}-x_{l}\right)\right] \\
\quad+\frac{i}{8} \sum_{\sigma \in \mathfrak{E}_{4}}\left[i E_{F}\left(x_{i}-x_{j}\right)\right]\left[i E_{F}\left(x_{j}-x_{k}\right)\right]\left[i E_{F}\left(x_{k}-x_{l}\right)\right]\left[i E_{F}\left(x_{l}-x_{i}\right)\right] \\
\quad+\frac{i}{4} \sum_{\sigma \in \mathfrak{E}_{4}}\left[i E_{F}\left(x_{i}-x_{k}\right)\right]\left[i E_{F}\left(x_{i}-x_{l}\right)\right]\left[i E_{F}\left(x_{k}-x_{l}\right)\right]\left[i E_{F}\left(x_{j}-x_{k}\right)\right] \\
\quad+\left[i E_{F}\left(x_{j}-x_{l}\right)\right] \\
\quad+i \prod_{1 \leqq j<k \leqq 4}\left[i E_{F}\left(x_{j}-x_{k}\right)\right] .
\end{aligned}
$$

Graphically, this corresponds to a sum of the subsequent diagrams

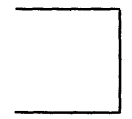

(1)

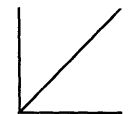

(2)

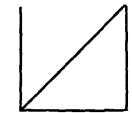

(3)

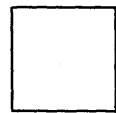

(4)

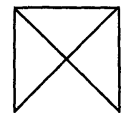

(5)

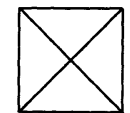

(6)

We shall show that the criterion of minimal singularity remains indeed applicable since the contribution from the points where $x_{1}^{0}=x_{2}^{0}=x_{3}^{0}=x_{4}^{0}$ to $\mathscr{R} e \tau_{f}\left(x_{1}^{0}, \ldots, x_{4}^{0}\right)$ can still be separated from the rest. This separability can be established although we do not know whether

$$
\mathscr{F}_{x_{1}, \ldots, x_{4}}\left\{\mathscr{R} e \tau\left(x_{1}, \ldots, x_{4}\right)\right\}\left(p_{1}, \ldots, p_{4}\right)
$$

decreases in some direction in momentum space. (It does certainly not decrease if only a subset of the invariant momenta $\left(p_{i}+p_{j}\right)^{2}$ grows beyond all bounds such that all partial sums of the momenta are timelike. However, a decrease when blowing up an arbitrary totally time-like configuration of the momenta has not been ruled out.) By requiring the absence of singular contributions from the points where $x_{1}^{0}=x_{2}^{0}=x_{3}^{0}=x_{4}^{0}$ to $\mathscr{R} e \tau_{f}\left(x_{1}^{0}, \ldots, x_{4}^{0}\right)$ we obtain a particular least singular definition of $\mathscr{R} e \tau_{f}$. The individual definitions for every real sufficiently smooth spatial test function can be shown to derive from one common, uniquely determined Lorentz invariant definition of $\mathscr{R} \tau\left(x_{1}, \ldots, x_{4}\right)$ as the 
corresponding spatial averages. By adding to it the imaginary part $\operatorname{Im} \tau\left(x_{1}, \ldots, x_{4}\right)$ uniquely determined by unitarity we are led to a least singular definition of $\tau\left(x_{1}, \ldots, x_{4}\right)$. There is no arbitrariness left.

This paper is organized as follows:

In Section III we study the behavior of quadratic forms with parameter dependent coefficients raised to some complex power like

$$
\left[\sum_{j=1}^{3} \sum_{k=1}^{3} a_{j k}\left(\theta_{1}, \theta_{2}\right) p_{j} \cdot p_{k} \pm i O\right]^{\mu}
$$

in $\theta_{1}, \theta_{2}$ and $\mu$ where the quadratic form may degenerate for certain values of $\theta_{1}$ and $\theta_{2}$, a prerequisite to the subsequent discussion. In Section III we introduce auxiliary amplitudes $3_{r}\left(x_{1}, \ldots, x_{4} ; \gamma\right)$ and $3\left(x_{1}, \ldots, x_{4}\right)$ and show how they are related to the two and three point Green's functions. None of these auxiliary amplitudes provides an admissable definition for the connected part of the time-ordered vacuum expectation value of four exponentials. However, the deficiences of 3 have a relatively simple form. They can be made good by adding the deficiency amplitudes $3\left(x_{1}, \ldots, x_{4}\right)$ introduced and studied in Section IV. Whereas the dependence of $\tilde{\mathfrak{Z}}\left(p_{1}, \ldots, p_{4}\right)=\mathscr{F}_{x_{1} \ldots x_{4}}\left\{\mathfrak{Z}\left(x_{1}, \ldots, x_{4}\right)\right\}\left(p_{1}, \ldots, p_{4}\right)$ on the momenta $p_{1}, \ldots, p_{4}$ is very complicated, the dependence of $\tilde{z}\left(p_{1}, \ldots, p_{4}\right)=\mathscr{F}_{x_{1} \ldots x_{4}}\left\{3\left(x_{1}, \ldots, x_{4}\right)\right\}\left(p_{1}, \ldots, p_{4}\right)$ on the momenta is of the same simple nature as that of the superpropagator. On the other hand, whereas it is relatively easy to control the asymptotic behavior of $\mathscr{R} e \tilde{\mathfrak{Z}}\left(p_{1}, \ldots, p_{4}\right)$ in the sector where all momenta and their partial sums are time-like this is not at all easy for $\mathscr{R} e \tilde{\mathfrak{z}}\left(p_{1}, \ldots, p_{4}\right)$.

In Section $\mathrm{V}$ the most general definition of $\tau\left(x_{1}, \ldots, x_{4}\right)$ is given and the structure of $\mathscr{R} e \tau_{f}\left(x_{1}^{0}, \ldots, x_{4}^{0}\right)$ is examined in regard to the separability property. Finally, among all admissable definitions we choose the least singular one as the definition that leads to the simplest dynamics associated with the given classical Lagrangian.

We use the notation of Ref. [2] and [7]. The spaces $\mathfrak{C}_{1 / 3}\left(\mathbb{R}^{l}\right)$ and $\mathfrak{C}_{1 / 3}^{\prime}\left(\mathbb{R}^{l}\right)$ denote the images of the spaces $\mathfrak{M}_{1 / 3}\left(\mathbb{R}^{l}\right)$ and $\mathfrak{M}_{1 / 3}^{\prime}\left(\mathbb{R}^{l}\right)$ respectively under Fourier transformation (cf. [2]).

\section{Powers of Parameter Depending Quadratic Forms}

In this section we shall investigate the behavior of powers $[P \pm i O]^{\mu}$ of quadratic forms whose coefficients depend on parameters:

$$
\begin{aligned}
& P=P\left(\{q\} ; \theta_{1}, \theta_{2}\right)=\sum_{j=1}^{3} \sum_{k=1}^{3} a_{j k}\left(\theta_{1}, \theta_{2}\right) q_{j} \cdot q_{k}, \\
& q_{j} \in \mathbb{R}^{4}, \quad \theta_{1}, \theta_{2} \in I=[0,1], \quad \mathscr{R} e \mu>-6,
\end{aligned}
$$


in particular for

$$
a_{j k}\left(\theta_{1}, \theta_{2}\right)= \begin{cases}b_{1}\left(\theta_{1}, \theta_{2}\right), & \text { if } j=k=1 \\ \theta_{2} b_{2}\left(\theta_{1}, \theta_{2}\right), & \text { if } j=k=2 \\ \theta_{1} \theta_{2} b_{3}\left(\theta_{1}, \theta_{2}\right), & \text { if } j=k=3 \\ 0 & \text { otherwise }\end{cases}
$$

Here, $b_{j}\left(\theta_{1}, \theta_{2}\right) j=1,2,3$ are negative, infinitely differentiable functions on the square $I \times I$.

From partial Fourier transformation with respect to the variables $q_{j}$ it is seen that the distribution-valued function (of $\theta_{1}$ and $\theta_{2}$ ) $[P \pm i O]^{\mu}$ is infinitely differentiable with respect to $\theta_{1}$ and $\theta_{2}$ as long as the quadratic form is not degenerate i.e. away from $\theta_{1}=0$ or $\theta_{2}=0$.

For $\mathscr{R} e \mu>-6$ the behavior of $[P \pm i O]^{\mu}$ as $\theta_{j} j=1,2$ approach the left end of $I$ is given by

$$
\begin{aligned}
{[P \pm i O]^{\mu}=} & F_{\mu}^{ \pm}\left(\{q\} ; \theta_{1}, \theta_{2}\right)+\theta_{2}^{2+\mu} G_{\mu}^{ \pm}\left(\{q\} ; \theta_{1}, \theta_{2}\right) \\
& +\theta_{2}^{2+\mu} \theta_{1}^{4+\mu} H_{\mu}^{ \pm}\left(\{q\} ; \theta_{1}, \theta_{2}\right)
\end{aligned}
$$

where the distribution-valued functions $F_{\mu}^{ \pm}, \ldots, H_{\mu}^{ \pm}$have the following properties: for $\theta_{j} \in I, G_{\mu}^{ \pm}$and $F_{\mu}^{ \pm}$are infinitely differentiable in $\theta_{1}$ and $\theta_{1}, \theta_{2}$ respectively;

$$
H_{\mu}^{ \pm},\left\{\frac{\partial^{m}}{\partial \theta_{1}^{m}} G_{\mu}^{ \pm}\right\}_{m=0,1, \ldots}\left(\left\{\frac{\partial^{m}}{\partial \theta_{1}^{m}} \frac{\partial^{n}}{\partial \theta_{2}^{n}} F_{\mu}^{ \pm}\right\}_{\substack{m=0,1, \ldots \\ n=0,1, \ldots}}\right)
$$

are continuous and bounded as $\theta_{j} j=1,2$ vary over the interval $I$.

$F_{\mu}^{ \pm}, \ldots, H_{\mu}^{ \pm}$depend analytically on $\mu$ in $\mathscr{R} e \mu>-6$. (For $\mu=-1$ and -2 the factors $\theta_{2}^{2+\mu}$ and $\theta_{1}^{4+\mu}$ have to be replaced by $\theta_{2}^{2+\mu} \ln \theta_{2}$ and $\theta_{1}^{4+\mu} \ln \theta_{1}$, for $\mu=-3$ and -4 the factor $\theta_{1}^{4+\mu}$ by $\theta_{1}^{4+\mu} \ln \theta_{1}$.)

Moreover, the limit of $[P \pm i \varepsilon]^{\mu}$ as $\varepsilon$ tends to +0 exists for $\theta_{j} \in \stackrel{\circ}{I}$, $j=1,2$ and is equal to $[P \pm i O]^{\mu}$ provided that $\mathscr{R} e \mu$ is larger than -6 :

$$
\lim _{\varepsilon \downarrow 0}[P \pm i \varepsilon]^{\mu}=[P \pm i O]^{\mu}
$$

Next, we turn to the asymptotic behavior in $\mu$ of the distribution $[P \pm i O]^{\mu}$ and of the function $[P \pm i \varepsilon]^{\mu}$ for $\mathscr{R} e \mu>-6$. To this end, we use the formula

$$
f(x)=\sum_{n=0}^{N} \frac{x^{n}}{n !} f^{(n)}(0)+\frac{1}{N !} \int_{0}^{1} d v(1-v)^{N} \frac{\partial^{N+1}}{\partial v^{N+1}} f(v x), f \in C^{N+1}\left(\mathbb{R}^{1}\right)
$$

for $f(x)=\left[b_{1}\left(\theta_{1}, \theta_{2}\right) q_{1}^{2}+\theta_{2} b_{2}\left(\theta_{1}, \theta_{2}\right) q_{2}^{2}+x \theta_{2} b_{3}\left(\theta_{1}, \theta_{2}\right) q_{3}^{2}+i_{0}^{\varepsilon}\right]^{\mu}$ with $3+\mathscr{R} e \mu \leqq N<4+\mathscr{R} e \mu$ in $\mathscr{R} e \mu>-4$. After having set $x$ equal to $\theta_{1}$ we 
estimate the resulting expansion term by term. In this way we obtain the following result:

There is a positive constant $M$ such that the set of distributions

$$
\begin{gathered}
\left\{\left(M\left[1+\sum_{j=1}^{3} \sum_{v=0}^{3}\left|q_{j, v}\right|^{2}\right]\left[1+\frac{(\mathscr{I} m \mu)^{2}}{(\mathscr{R} e \mu+6)^{2}}\right]^{1 / 2}\right)^{-\mathscr{R}_{e} \mu-6}\right. \\
\left.e^{-\pi\left|\mathscr{I}_{m} \mu\right|}[P \pm i \varepsilon]^{\mu} / \mathscr{R}_{e} \mu>-6 ; 0 \leqq \varepsilon \leqq 1\right\}
\end{gathered}
$$

is bounded in the topology of $\mathscr{S}^{\prime}\left(\mathbb{R}^{12}\right)$.

Now, we apply these pieces of information to the powers of quadratic forms that actually occur in the momentum space expression for the connected part of the time-ordered vacuum expectation value of four exponentials. We restrict ourselves to the discussion of the powers of quadratic forms corresponding to the diagrams (4), (5), and (6), while the reader is referred to Ref. [2] for a discussion of those powers that correspond to the remaining diagrams (1), (2), and (3).

With Speer [7] we define the determinants

i) $C_{4}^{\varrho}(\{\alpha\})$ and $D_{4}^{\varrho}(\{\alpha\} ;\{p\})$ for the diagram (4), where $\varrho \in \mathfrak{P}_{41} \cup \mathfrak{P}_{42}$ denotes a permutation from the sets

$$
\mathfrak{P}_{41}=\left\{\left(\begin{array}{l}
1 \\
1
\end{array}\right)\left(\begin{array}{lll}
2 & 3 & 4 \\
2 & 3 & 4
\end{array}\right),\left(\begin{array}{l}
1 \\
1
\end{array}\right)\left(\begin{array}{lll}
2 & 3 & 4 \\
3 & 2 & 4
\end{array}\right),\left(\begin{array}{l}
1 \\
1
\end{array}\right)\left(\begin{array}{lll}
2 & 3 & 4 \\
2 & 4 & 3
\end{array}\right)\right\}, \quad \mathfrak{P}_{42}=\phi
$$

and where the correspondence of the Feynman parameters $\alpha_{\varrho(1)}, \ldots, \alpha_{\varrho(4)}$ and the internal lines is shown in the following diagram

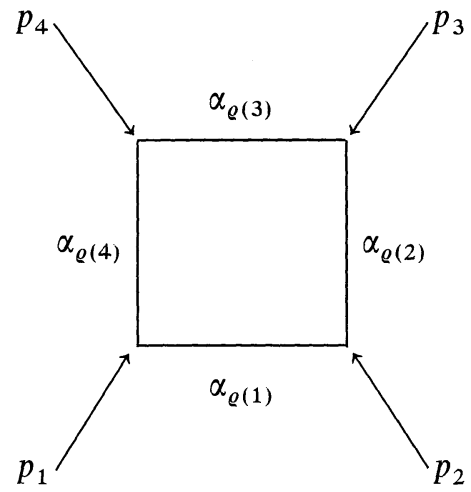

ii) $C_{5}^{\varrho}(\{\alpha\})$ and $D_{5}^{\varrho}(\{\alpha\} ;\{p\})$ for the diagram (5), where $\varrho \in \mathfrak{P}_{51} \cup \mathfrak{P}_{52}$ denotes a permutation from the sets

$$
\begin{aligned}
& \mathfrak{P}_{51}=\left\{\left(\begin{array}{l}
1 \\
1
\end{array}\right) \hat{\varrho} / \varrho \in \Xi_{4},(\varrho(3), \hat{\varrho}(4)) \neq \begin{array}{l}
(4,5) \\
(5,4)
\end{array}\right\} \cup\left\{\left(\begin{array}{ll}
1 & 2 \\
2 & 1
\end{array}\right) \hat{\varrho} / \varrho \in \Xi_{3}, \hat{\varrho}(5) \neq 3\right\} \\
& \mathfrak{P}_{52}=\left\{\left(\begin{array}{l}
1 \\
1
\end{array}\right) \hat{\varrho} / \hat{\varrho} \in \Xi_{4},(\hat{\varrho}(3), \hat{\varrho}(4))=\begin{array}{l}
(4,5) \\
(5,4)
\end{array}\right\} \cup\left\{\left(\begin{array}{ll}
1 & 2 \\
2 & 1
\end{array}\right) \hat{\varrho} / \varrho \in \Xi_{3}, \hat{\varrho}(5)=3\right\}
\end{aligned}
$$


and where the correspondence of the Feynman parameters $\alpha_{\varrho(1)}, \ldots, \alpha_{\varrho(5)}$ and the internal lines is shown in the following diagram

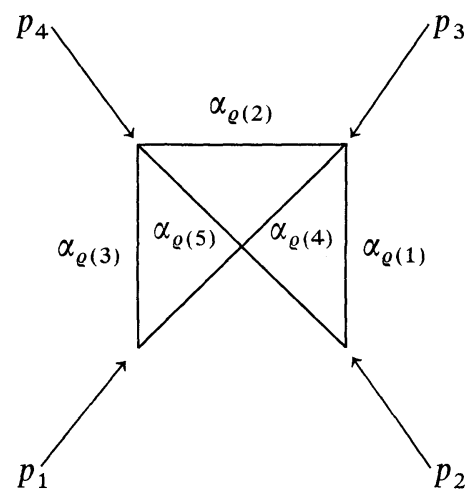

iii) $C_{6}^{\varrho}(\{\alpha\})$ and $D_{6}^{\varrho}(\{\alpha\} ;\{p\})$ for the diagram (6), where $\varrho \in \mathfrak{P}_{61} \cup \mathfrak{P}_{62}$ denotes a permutation from the sets

$$
\begin{aligned}
& \mathfrak{P}_{61}=\left\{\left(\begin{array}{l}
1 \\
1
\end{array}\right) \hat{\varrho} / \varrho \in \mathfrak{\Xi}_{4}, \hat{\varrho}(5) \neq 3\right\} \cup\left\{\left(\begin{array}{l}
1 \\
1
\end{array}\right)\left(\begin{array}{ll}
2 & 3 \\
3 & 2
\end{array}\right) \hat{\varrho} / \hat{\varrho} \in \Xi_{3}\right\} \\
& \mathfrak{P}_{62}=\left\{\left(\begin{array}{l}
1 \\
1
\end{array}\right)\left(\begin{array}{l}
2 \\
2
\end{array}\right) \hat{\varrho} / \hat{\varrho} \in \mathfrak{\Xi}_{4}, \hat{\varrho}(5)=3\right\}
\end{aligned}
$$

and where the correspondence of the Feynman parameters $\alpha_{\varrho(1)}, \ldots, \alpha_{\varrho(6)}$ and the internal lines is shown in the following diagram

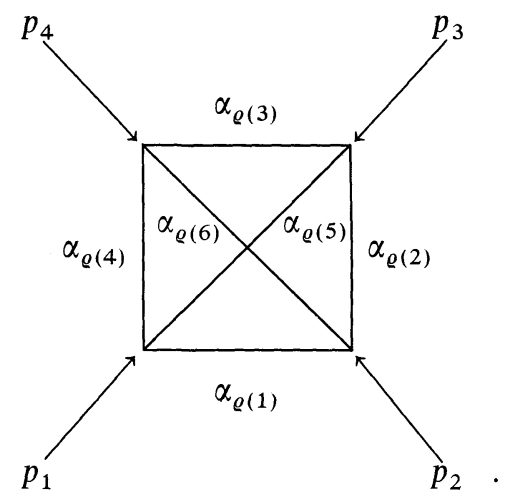

We set $\alpha_{j}=t_{a} \ldots t_{j} j=1, \ldots, a a=4,5,6$ where $t_{j} \in I$ for $j=1, \ldots, a-1$ and $t_{a} \in[0,+\infty[$.

The quotients $C_{a}^{\varrho}(\{\alpha\}) / \alpha_{a} \ldots \alpha_{4}$ for $\varrho \in \mathfrak{P}_{a 1}$ and $C_{a}^{\varrho}(\{\alpha\}) / \alpha_{a} \ldots \hat{\alpha}_{4} \alpha_{3}$ for $\varrho \in \mathfrak{P}_{a 2}$ are polynomials in $t_{1}, \ldots, t_{a-1}$, independent of $t_{a}$ and positive for 
$t_{j} \in I \quad j=1, \ldots, a-1$. The quotients $D_{a}^{\varrho}(\{\alpha\} ;\{p\}) / \alpha_{a} \ldots \alpha_{3}, \varrho \in \mathfrak{P}_{a 1} \cup \mathfrak{P}_{a 2}$, are quadratic forms in the moments $p_{1}, \ldots, p_{4} / \sum_{I}^{4} p_{m}=0$ with coefficients that depend polynomially on the parameters $t_{1}, \ldots, t_{a-1}$ and are independent of $t_{a}$. We consider the following parameter dependent distributions

and

$$
\delta\left(\sum_{1}^{4} p_{m}\right)\left[-\frac{D_{a}^{\varrho}(\{\alpha\} ;\{p\}) / \alpha_{a} \ldots \alpha_{3}}{C_{a}^{e}(\{\alpha\}) / \alpha_{a} \ldots \alpha_{4}} \pm i \varepsilon\right]^{\mu} \text { for } \varrho \in \mathfrak{P}_{a 1}
$$

$$
\delta\left(\sum_{1}^{4} p_{m}\right)\left[-\frac{D_{a}^{e}(\{\alpha\} ;\{p\}) / \alpha_{a} \ldots \alpha_{3}}{C_{a}^{e}(\{\alpha\}) / \alpha_{a} \ldots \hat{\alpha}_{4} \alpha_{3}} \pm i \varepsilon\right]^{\mu} \text { for } \varrho \in \mathfrak{P}_{a 2}
$$

with $\varepsilon \geqq 0, t_{j} \in I j=1, \ldots, a-1$ and $\mathscr{R} e \mu>-6$ and where the GelfandShilov prescription is taken for $\varepsilon=0$ [8].

For any permutation $\varrho$ from the set $\mathfrak{P}_{a 1} \cup \mathfrak{P}_{a 2}$ there exists a nonsingular linear transformation $T_{a}^{e}(\{t\})$, infinitely differentiable with respect to $t_{j} j=1, \ldots, a-1$,

$$
\left(\begin{array}{l}
q_{1}^{o}\left(t_{1}, \ldots, t_{a-1} ;\{p\}\right) \\
q_{2}^{o}\left(t_{1}, \ldots, t_{a-1} ;\{p\}\right) \\
q_{3}^{o}\left(t_{1}, \ldots, t_{a-1},\{p\}\right) \\
p_{1}+p_{2}+p_{3}+p_{4}
\end{array}\right)=T_{a}^{Q}\left(t_{1}, \ldots, t_{a-1}\right)\left(\begin{array}{l}
p_{1} \\
p_{2} \\
p_{3} \\
p_{4}
\end{array}\right)
$$

which diagonalizes the corresponding quadratic form such that the distributions (1) and ( $\left(1^{\prime}\right)$ take the shape

$$
\begin{gathered}
\delta\left(\sum_{1}^{4} p_{m}\right)\left[-\left(q_{1}^{\varrho}\right)^{2}-t_{2}\left(q_{2}^{\rho}\right)^{2}-t_{2} t_{1}\left(q_{3}^{\varrho}\right)^{2} \pm i \varepsilon\right]^{\mu} \\
\delta\left(\sum_{1}^{4} p_{m}\right)\left[-\left(q_{1}^{\varrho}\right)^{2}-t_{3} t_{2}\left(q_{2}^{\rho}\right)^{2}-t_{3} t_{2} t_{1}\left(q_{2}^{\rho}\right)^{2} \pm i \varepsilon\right]^{\mu}
\end{gathered}
$$

for $\varrho \in \mathfrak{P}_{a 1}$ and $\varrho \in \mathfrak{P}_{a 2}$ respectively.

By applying the previously established results about powers of parameter depending quadratic forms, we arrive at the following

Lemma 1. The behavior of the distributions (1) and $\left(1^{\prime}\right): \varepsilon \geqq 0$, for $\mathscr{R e} \mu>-6$ as functions of $t_{1}, \ldots, t_{a-1}$ is given by

and

$$
\begin{gathered}
F_{\mu, a, \varrho}^{ \pm \varepsilon}\left(t_{1}, \ldots, t_{a-1} ;\{p\}\right)+t_{2}^{2+\mu} G_{\mu, a, \varrho}^{ \pm \varepsilon}\left(t_{1}, \ldots, t_{a-1} ;\{p\}\right) \\
+t_{2}^{2+\mu} t_{1}^{4+\mu} H_{\mu, a, \varrho}^{ \pm \varepsilon}\left(t_{1}, \ldots, t_{a-1} ;\{p\}\right) \text { for } \varrho \in \mathfrak{P}_{a 1}
\end{gathered}
$$

$$
\begin{aligned}
& F_{\mu, a, \varrho}^{ \pm \varepsilon}\left(t_{1}, \ldots, t_{a-1} ;\{p\}\right)+\left(t_{3} t_{2}\right)^{2+\mu} G_{\mu, a, e}^{ \pm \varepsilon}\left(t_{1}, \ldots, t_{a-1} ;\{p\}\right) \\
& +\left(t_{3} t_{2}\right)^{2+\mu} t_{1}^{4+\mu} H_{\mu, a, \varrho}^{ \pm \varepsilon}\left(t_{1}, \ldots, t_{a-1} ;\{p\}\right) \text { for } \varrho \in \mathfrak{P}_{a 2}
\end{aligned}
$$


respectively. Here, the distribution-valued functions $F_{\mu, a, \varrho}^{ \pm \varepsilon}, G_{\mu, a, \varrho}^{ \pm \varepsilon}$, and $H_{\mu, a, \varrho}^{ \pm \varepsilon}$ have the following properties: for $t_{j} \in I j=1, \ldots, a-1, F_{\mu, a, \varrho}^{ \pm \varepsilon}$ is an infinitely differentiable function; for $t_{j} \in I^{\circ} j=1, \ldots, a-1$, also $G_{\mu, a, \varrho}^{ \pm \varepsilon}$ and $H_{\mu, a, \varrho}^{ \pm \varepsilon}$ are infinitely differentiable functions, while for $t_{j} \in I j=1, \ldots, a-1$ $G_{\mu, a, \varrho}^{ \pm \varepsilon}$ remains infinitely differentiable with respect to

$$
\left\{\begin{array}{lll}
t_{1}, t_{3}, \ldots, t_{a-1} & \text { if } & \varrho \in \mathfrak{P}_{a 1} \\
t_{1}, t_{4}, \ldots, t_{a-1} & \text { if } & \varrho \in \mathfrak{P}_{a 2}
\end{array}\right\} \quad \text { and } H_{\mu, a, \varrho}^{ \pm \varepsilon}
$$

infinitely differentiable with respect to

$$
\left\{\begin{array}{lll}
t_{3}, \ldots, t_{a-1} & \text { if } & \varrho \in \mathfrak{P}_{a 1} \\
t_{4}, \ldots, t_{a-1} & \text { if } & \varrho \in \mathfrak{P}_{a 1}
\end{array}\right\} .
$$

$\left\{\frac{\partial^{m}}{\partial t_{1}^{m}} G_{\mu, a, \varrho}^{ \pm \varepsilon}\right\}_{m=0,1, \ldots}$ and $H_{\mu, a, \varrho}^{ \pm \varepsilon}$ are continuous (hence bounded) functions over the product of the closed unit intervals $I . F_{\mu, a, \varrho}^{ \pm \varepsilon}, G_{\mu, a, \varrho}^{ \pm \varepsilon}$, and $H_{\mu, a, \varrho}^{ \pm \varepsilon}$ depend analytically on $\mu$ in the domain $\mathscr{R} e \mu>-6$. (Similar replacements as before have to be made for $\mu=-1, \ldots$, - 4.) Moreover, the limit of the functions (1) and (1') as $\varepsilon$ tends to +0 exists for $\mathscr{R e} \mu>-6, t_{j} \in \stackrel{\circ}{I} j=1, \ldots$ $\ldots, a-1$ and coincides with the Gelfand-Shilov prescription:

$$
\lim _{\varepsilon \downarrow 0} \delta\left(\sum_{1}^{4} p_{m}\right)[\cdots \pm i \varepsilon]^{\mu}=\delta\left(\sum_{1}^{4} p_{m}\right)[\cdots \pm i O]^{\mu}
$$

Thus the distributions

$$
\begin{aligned}
& \delta\left(\sum_{1}^{4} p_{m}\right) Z_{a, \varrho, \varepsilon}^{\sigma \pm}\left(\{p\} ; s_{1}, \ldots, s_{a}\right)=\int_{0}^{1} d t_{a-1} t_{a-1}^{\left(s_{a}+2\right)-1} \ldots \int_{0}^{1} d t_{3} t_{3}^{s_{a}+\cdots+s_{4}+2(a-3)-1} \\
& \cdot \int_{0}^{1} d t_{2} t_{2}^{-\left(s_{2}+s_{1}\right)-1} \int_{0}^{1} d t_{1} t_{1}^{-s_{1}-1}\left[C_{a}^{\varrho}(\{\alpha\}) / \alpha_{a} \ldots \alpha_{4}\right]^{-2} \delta\left(\sum_{1}^{4} p_{m}\right) \\
& \cdot\left[-\frac{D_{a}^{\varrho}\left(\{\alpha\} ; p_{\sigma(1)}, \ldots, p_{\sigma(4)}\right) / \alpha_{a} \ldots \alpha_{3}}{C_{a}^{\varrho}(\{\alpha\}) / \alpha_{a} \ldots \alpha_{4}} \pm i \varepsilon\right]^{\sum_{1}^{a} s_{n}+2(a-3)} \text { if } \varrho \in \mathfrak{P}_{a 1}, \\
& \delta\left(\sum_{1}^{4} p_{m}\right) Z_{a, \varrho, \varepsilon}^{\sigma \pm}\left(\{p\} ; s_{1}, \ldots, s_{a}\right)=\int_{0}^{1} d t_{a-1} t_{a-1}^{\left(s_{a}+2\right)-1} \ldots \int_{0}^{1} d t_{4} t_{4}^{s_{a}+\cdots+s_{5}+2(a-4)-1} \\
& \quad \cdot \int_{0}^{1} d t_{3} t_{3}^{-\left(s_{3}+s_{2}+s_{1}\right)-3} \int_{0}^{1} d t_{2} t_{2}^{-\left(s_{2}+s_{1}\right)-1} \int_{0}^{1} d t_{1} t_{1}^{-s_{1}-1}\left[C_{a}^{\varrho}(\{\alpha\}) / \alpha_{a} \ldots \hat{\alpha}_{4} \alpha_{3}\right]^{-2} \\
& \left.\quad \cdot \delta\left(\sum_{1}^{4} p_{m}\right)\left[-\frac{D_{a}^{\varrho}\left(\{\alpha\} ; p_{\sigma(1)}, \ldots, p_{\sigma(4)}\right) / \alpha_{a} \ldots \alpha_{3}}{C_{a}^{\varrho}(\{\alpha\}) / \alpha_{a} \ldots \hat{\alpha}_{4} \alpha_{3}} \pm i \varepsilon\right]^{a}\right]_{1}^{\sum s_{n}+2(a-3)} \text { if } \varrho \in \mathfrak{P}_{a 2}\left(4^{\prime}\right)
\end{aligned}
$$


with $\sigma \in \Im_{4}$ and $0 \leqq \varepsilon \leqq 1$, unambiguously defined in

$$
\begin{gathered}
\left\{\left(s_{1}, \ldots, s_{a}\right) / \mathscr{R} e s_{1}<0, \mathscr{R} e s_{1}+\mathscr{R} e s_{2}<0, \sum_{n+1}^{a} \mathscr{R} e s_{m}>-2(a-n)\right. \\
\left.n=3, \ldots, a-1, \sum_{1}^{a} \mathscr{R} e s_{m}>-2(a-2)\right\}
\end{gathered}
$$

and

$$
\begin{gathered}
\left\{\left(s_{1}, \ldots, s_{a}\right) / \mathscr{R} e s_{1}<0, \mathscr{R} e s_{1}+\mathscr{R} e s_{2}<0, \mathscr{R} e s_{1}+\mathscr{R} e s_{2}+\mathscr{R} e s_{3}<-2,\right. \\
\left.\sum_{n+1}^{a} \mathscr{R} e s_{m}>-2(a-n) \quad n=4, \ldots, a-1, \sum_{1}^{a} \mathscr{R} e s_{m}>-2(a-2)\right\}
\end{gathered}
$$

respectively, can be analytically continued in $s_{1}, \ldots, s_{a}$ to a function meromorphic in $\Omega_{a}$

$$
\Omega_{a}=\left\{\left(s_{1}, \ldots, s_{a}\right) / \frac{\sum_{n+1}^{a} \mathscr{R} e s_{m}}{a-n}>-2 \quad n=0,1, \ldots, a-1\right\} .
$$

If we use the same symbol for the continued function we obtain the following assertion:

$\Gamma\left(-s_{2}-s_{1}\right)^{-1} \Gamma\left(-s_{1}\right)^{-1} \delta\left(\sum_{1}^{4} p_{m}\right) Z_{a, \varrho}^{\sigma \pm}\left(\{p\} ; s_{1}, \ldots, s_{a}\right)$ for $\varrho \in \mathfrak{P}_{a 1}$

and

$\Gamma\left(-s_{3}-s_{2}-s_{1}-2\right)^{-1} \Gamma\left(-s_{2}-s_{1}\right)^{-1} \Gamma\left(-s_{1}\right)^{-1} \delta\left(\sum_{1}^{4} p_{m}\right) Z_{a, \varrho, \varepsilon}^{\sigma \pm}\left(\{p\} ; s_{1}, \ldots, s_{a}\right)$

for $\varrho \in \mathfrak{P}_{a 2}$ are analytic in $\Omega_{a}$, the limits as $\varepsilon$ tends to +0 exist there and are equal to

and

$$
\Gamma\left(-s_{2}-s_{1}\right)^{-1} \Gamma\left(-s_{1}\right)^{-1} \delta\left(\sum_{1}^{4} p_{m}\right) Z_{a, \varrho}^{\sigma \pm}, 0\left(\{p\} ; s_{1}, \ldots, s_{a}\right)
$$

$\Gamma\left(-s_{3}-s_{2}-s_{1}-2\right)^{-1} \Gamma\left(-s_{2}-s_{1}\right)^{-1} \Gamma\left(-s_{1}\right)^{-1} \delta\left(\sum_{1}^{4} p_{m}\right) Z_{a, o, 0}^{\sigma \pm}\left(\{p\} ; s_{1}, \ldots, s_{a}\right)$

respectively.

Finally, we apply the results on the asymptotic behavior in $\mu$ of the powers $[P \pm i \varepsilon]^{\mu}$ established at the beginning of this section to the distributions (1) and $\left(1^{\prime}\right)$. Thereby we derive 
Lemma 2. There exists a positive constant $M$ such that the sets of distributions $B_{a_{1}}$ and $B_{a_{2}}$ are bounded (in the topology of $\mathscr{S}^{\prime}\left(\mathbb{R}^{16}\right)$ ) where

$$
\begin{aligned}
& B_{a 1}=\left\{\left(M\left[1+\sum_{m=1}^{4} \sum_{v=0}^{3}\left|p_{m, v}\right|^{2}\right]\left[1+\left(\frac{\sum_{1}^{a} \mathscr{I}_{m} s_{m}}{\sum_{1}^{a} \mathscr{R}_{e} s_{m}+12}\right)^{2}\right]^{1 / 2}\right]^{-\sum_{1}^{a} \mathscr{R}_{e} s_{m}-12}\right. \\
& \cdot\left[\prod_{n=1}^{2} e^{-\pi\left|\sum_{1}^{n} \mathscr{I}_{m} s_{m}\right|}\left|\Gamma\left(1+\sum_{1}^{n} s_{m}\right)^{-1}\right|\right] e^{-\pi\left|\sum_{1}^{a} \mathscr{I}_{m} s_{m}\right|} \Gamma\left(-s_{2}-s_{1}\right)^{-1} \Gamma\left(-s_{1}\right)^{-1} \\
& \cdot \delta\left(\sum_{1}^{4} p_{m}\right) Z_{a, \varrho, \varepsilon}^{\sigma \pm}\left(\{p\} ; s_{1}, \ldots, s_{a}\right) /\left(s_{1}, \ldots, s_{a}\right) \in \Omega_{a}, \sigma \in \mathfrak{S}_{4}, \\
& \left.\varrho \in \mathfrak{P}_{a, 1}, 0 \leqq \varepsilon \leqq 1\right\}
\end{aligned}
$$$$
B_{a 2}=\left\{\left(M\left[1+\sum_{m=1}^{4} \sum_{\nu=0}^{3}\left|p_{m, v}\right|^{2}\right]\left[1+\left(\frac{\sum_{1}^{a} \mathscr{I} m s_{m}}{\sum_{1}^{a} \mathscr{R} e s_{m}+12}\right)^{2}\right]^{1 / 2}\right)^{-\sum_{1}^{a} \mathscr{R} e s_{m}-12}\right.
$$$$
\cdot\left[\prod_{n=1}^{3} e^{-\pi \mid \sum_{1}^{n} \mathscr{I}_{m} s_{m}}|| \Gamma\left(|2 n-3|+\sum_{1}^{n} s_{m}\right)^{-1} \mid\right] e^{-\pi\left|\sum_{1}^{a} \mathscr{I}_{m} s_{m}\right|}
$$$$
\cdot \Gamma\left(-s_{3}-s_{2}-s_{1}-2\right)^{-1} \Gamma\left(-s_{2}-s_{1}\right)^{-1} \Gamma\left(-s_{1}\right)^{-1} \delta\left(\sum_{1}^{4} p_{m}\right)
$$$$
\left.\cdot Z_{a, \varrho, \varepsilon}^{\sigma \pm}\left(\{p\} ; s_{1}, \ldots, s_{a}\right) /\left(s_{1}, \ldots, s_{a}\right) \in \Omega_{a}, \sigma \in \Im_{4}, \varrho \in \mathfrak{P}_{a 2}, 0 \leqq \varepsilon \leqq 1\right\} .
$$

\section{Auxiliary Amplitudes}

\section{A. Unitarity and Locality Relations}

We define auxiliary amplitudes $\underset{\left.\mathcal{F}_{r}\right)}{\mathcal{3}_{r}}\left(p_{1}, \ldots, p_{4} ; \gamma\right) r= \pm 1, \pm 3, \pm 5, \pm 7$ for sufficiently large real values of $\gamma$, i.e. $\gamma>2 r+3$ by

$$
\widetilde{\widetilde{Z}}_{r}\left(p_{1}, \ldots, p_{4} ; \gamma\right)=\sum_{a=1}^{6} \widetilde{(\widetilde{3})}_{a, r}\left(p_{1}, \ldots, p_{4} ; \gamma\right)
$$


with

$\left(\frac{1}{3}\right)$

$\widetilde{\overline{3}}_{1, r}\left(p_{1}, \ldots, p_{4} ; \gamma\right)$

$$
\begin{aligned}
= & \delta\left(\sum_{1}^{4} p_{m}\right) \sum_{\sigma \in \mathbb{S}_{4}} \frac{\lambda^{6}}{2}\left(\frac{\pi}{4}\right)^{2} \int_{S-i \infty}^{S} \cdots \int_{n=1}^{S+i \infty}\left[\prod_{n=1}^{3} \frac{d s_{n}}{2 \pi i} e^{i \pi r s_{n}} \frac{\Gamma\left(-\gamma\left(1+s_{n}\right)\right) \Gamma\left(-s_{n}\right)}{\Gamma\left(3+s_{n}\right)}\right] \\
& {\left[\frac{\lambda}{4}\left(-p_{i}^{2}(\overline{-}) i O\right)\right]^{s_{3}}\left[\frac{\lambda}{4}\left(-\left(p_{i}+p_{j}\right)^{2} \overline{(\mp)} i O\right)\right]^{s_{2}}\left[\frac{\lambda}{4}\left(-\left(p_{i}+p_{j}+p_{k}\right)^{2} \overline{(-)} i O\right)\right]^{s_{1}}, }
\end{aligned}
$$

$\widetilde{\widetilde{3}}_{2, r}\left(p_{1}, \ldots, p_{4} ; \gamma\right)$

$$
\begin{aligned}
& =\delta\left(\sum_{1}^{4} p_{m}\right) \sum_{\sigma \in \mathscr{E}_{4}} \frac{\lambda^{6}}{6}\left(\frac{\pi}{4}\right)^{2} \int_{S-i \infty}^{S+i \infty}\left[\prod_{n=1}^{3} \frac{d s_{n}}{2 \pi i} e^{i \pi r s_{n}} \frac{\Gamma\left(-\gamma\left(1+s_{n}\right)\right) \Gamma\left(-s_{n}\right)}{\Gamma\left(3+s_{n}\right)}\right] \\
& {\left[\frac{\lambda}{4}\left(-p_{j}^{2}(\bar{H} i O)\right]^{s_{3}}\left[\frac{\lambda}{4}\left(-p_{k}^{2}(\bar{F}) i O\right)\right]^{s_{2}}\left[\frac{\lambda}{4}\left(-p_{l}^{2}(\bar{F}) i O\right)\right]^{s_{1}}\right. \text {, }}
\end{aligned}
$$

$\widetilde{\overline{3}}_{3, r}\left(p_{1}, \ldots, p_{4} ; \gamma\right)$

$$
\begin{aligned}
= & -\delta\left(\sum_{1}^{4} p_{m}\right) \sum_{\sigma \in \mathfrak{S}_{4}} \frac{\lambda^{6}}{2}\left(\frac{\pi}{4}\right)^{2} \int_{S-i \infty}^{S+i \infty}\left[\prod_{n=1}^{4} \frac{d s_{n}}{2 \pi i} e^{i \pi r s_{n}} \frac{\Gamma\left(-\gamma\left(1+s_{n}\right)\right)}{\Gamma\left(3+s_{n}\right)}\right] \\
& \cdot \Gamma\left(-s_{4}\right)\left[\frac{\lambda}{4}\left(-p_{l}^{2} \text { (Ғ } i O\right)\right]^{s_{4}}\left(\frac{\lambda}{4}\right)^{\sum_{1} s_{n}+2} \Gamma\left(-\sum_{1}^{3} s_{n}-2\right) \\
& \cdot \sum_{\varrho \in \mathfrak{S}_{3}} T_{0}^{\varrho( \pm)}\left(p_{k}, p_{i},-p_{i}-p_{k} ; s_{1}, \ldots, s_{3}\right),
\end{aligned}
$$

$\widetilde{\widetilde{3}}_{a, r}\left(p_{1}, \ldots, p_{4} ; \gamma\right)$

$$
\begin{aligned}
& =\delta\left(\sum_{1}^{4} p_{m}\right)(-1)^{a+1} 4^{6}\left(\frac{\lambda}{4}\right)^{2 a}\left(\frac{\pi}{4}\right)^{2} \sum_{\sigma \in \subseteq_{4}} \int_{S-i \infty}^{S} \cdots \int\left[\prod_{n=1}^{+i \infty} \frac{d s_{n}}{2 \pi i}\left(\frac{\lambda}{4}\right)^{s_{n}}\right. \\
& \left.\cdot e^{i \pi r s_{n}} \frac{\Gamma\left(-\gamma\left(1+s_{n}\right)\right)}{\Gamma\left(3+s_{n}\right)}\right] \Gamma\left(-\sum_{1}^{a} s_{n}-2(a-3)\right) \sum_{\varrho \in \mathfrak{P}_{a 1} \cup \mathfrak{P}_{a 2}} Z_{a, \varrho}^{\sigma( \pm)}\left(\{p\} ; s_{1}, \ldots, s_{a}\right)
\end{aligned}
$$

for $a=4,5,6 . S$ is a real number between -2 and -1 . With the help of Lemma 2 and Stirling's formula it can be shown that for $\gamma$ real and larger than seventeen the above integrals exist and define distributions contained in the class $\mathfrak{M}_{1 / 3}^{\prime}\left(\mathbb{R}^{16}\right)$ :

$$
\widetilde{\widetilde{3}}_{r}\left(p_{1}, \ldots, p_{4} ; \gamma\right) \in \mathfrak{M}_{1 / 2}^{\prime}\left(\mathbb{R}^{16}\right) \text {. }
$$

By swinging the $s_{n}$-contours around the real axis from -1 to $+\infty$ in the same way as it has done in Ref. [2] one proves the existence of a constant $\delta>0$ such that $\widetilde{\overline{3}}_{r}\left(p_{1}, \ldots, p_{4} ; \gamma\right) r= \pm 1, \ldots, \pm 7$ are analytic functions of $\gamma$ 
in the chisel shaped region

$$
W_{\delta, 4}=\left\{\gamma=\gamma_{1}+i \gamma_{2} / \gamma_{1}>1,\left|\gamma_{2}\right|<\operatorname{Min}\left[\delta, \frac{\pi}{16}\left(\gamma_{1}-1\right)\right]\right\}
$$

with values in $\mathfrak{M}_{1 / 3}^{\prime}\left(\mathbb{R}^{16}\right)$ and that the limits

$$
\widetilde{\overline{3}}_{r}\left(p_{1}, \ldots, p_{4}\right)=\lim _{\gamma \rightarrow 1, \gamma \in W_{\delta, 4}} \widetilde{\widetilde{3}}_{r}\left(p_{1}, \ldots, p_{4} ; \gamma\right)
$$

exist in $\mathfrak{M}_{1 / 3}^{\prime}\left(\mathbb{R}^{16}\right)$.

Next, we want to show that for $\gamma$ real and larger than seventeen the Fourier transform of $\widetilde{\widetilde{3}_{r}}\left(p_{1}, \ldots, p_{4} ; \gamma\right)$ is related to the product

$$
\prod_{1 \leqq i<j \leqq 4}\left[1 \uplus i E_{F(\bar{F}), r}\left(x_{i}-x_{j} ; \gamma\right)\right]
$$

by the following equations

$$
\begin{aligned}
\mathcal{Z}_{r}\left(x_{1}, \ldots, x_{4} ; \gamma\right) & =\overline{\mathscr{F}}_{p_{1}, \ldots, p_{4}}\left\{\tilde{\mathcal{Z}}_{r}\left(p_{1}, \ldots, p_{4} ; \gamma\right)\right\}\left(x_{1}, \ldots, x_{4}\right) \\
& =i\left(\prod_{1 \leqq i<j \leqq 4}\left[1+i E_{F, r}\left(x_{i}-x_{j} ; \gamma\right)\right]\right)_{\text {conn. }} \\
\overline{\mathcal{Z}}_{r}\left(x_{1}, \ldots, x_{4} ; \gamma\right) & =\overline{\mathscr{F}}_{p_{1}, \ldots, p_{4}}\left\{\widetilde{\bar{Z}}_{r}\left(p_{1}, \ldots, p_{4} ; \gamma\right)\right\}\left(x_{1}, \ldots, x_{4}\right) \\
& =-i\left(\prod_{1 \leqq i<j \leqq 4}\left[1-i E_{\bar{F}, r}\left(x_{i}-x_{j} ; \gamma\right)\right]\right)_{\text {conn. }} .
\end{aligned}
$$

The ultra distributions $E_{F(\bar{F}), r}(x ; \gamma)$ occurring on the right hand sides of the above equations as well as the corresponding ultra distributions $E_{r}^{( \pm)}(x ; \gamma)$ were defined in Ref. [2]. From the results established there, we infer that

a) $E_{F(\bar{F}), r}(x ; \gamma)$ and $E_{r}^{( \pm)}(x ; \gamma)$ are analytic functions of $\gamma$ in $W_{\delta, 4}$ with values in $\widetilde{C}_{1 / 3}^{\prime}\left(\mathbb{R}^{4}\right)$,

b) the limits

$$
\lim _{\gamma \rightarrow 1, \gamma \in W_{\delta, 4}} E_{F(\bar{F}), r}(x ; \gamma) \text { and } \lim _{\gamma \rightarrow 1, \gamma \in W_{\delta, 4}} E_{r}^{( \pm)}(x ; \gamma)
$$

exist in $\mathfrak{C}_{1 / 3}^{\prime}\left(\mathbb{R}^{4}\right)$ and are equal to

and

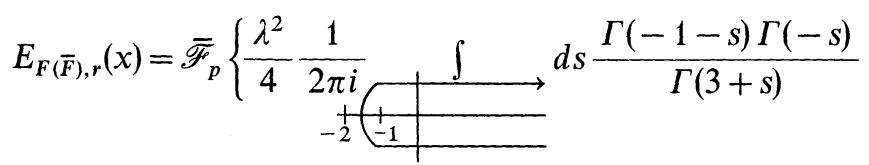

$$
\begin{aligned}
& \left.\cdot e^{i \pi r s}\left[\frac{\lambda}{4}\left(-p^{2} \mp i O\right)\right]^{s}\right\}(x)
\end{aligned}
$$

$$
E^{( \pm)}(x)=\overline{\mathscr{F}}_{p}\left\{-2 \pi i \lambda \delta_{ \pm}\left(p^{2}\right)-2 \pi i \frac{\lambda^{2}}{4} \Theta\left( \pm p_{0}\right) \Theta\left(p^{2}\right) g\left(\frac{\lambda}{4} p^{2}\right)\right\}(x)
$$


respectively where

$$
g(w)=\sum_{m=0}^{\infty} \frac{w^{m}}{m !(m+1) !(m+2) !},
$$

c) $i E_{F, r}(x ; \gamma)$ and $-i E_{\bar{F}, r}(x ; \gamma)$ are time ordered "functions" in the following sense

$$
\begin{array}{r}
i E_{F, r}(x ; \gamma)=\left\{\begin{array}{lll}
i E_{r}^{(+)}(x ; \gamma) & \text { for } & x^{0}>0 \\
i E_{r}^{(+)}(-x ; \gamma) & \text { for } & x^{0}<0
\end{array}\right. \\
-i E_{\bar{F}, r}(x ; \gamma)=\left\{\begin{array}{lll}
i E_{r}^{(+)}(-x ; \gamma) & \text { for } & x^{0}>0 \\
i E_{r}^{(+)}(x ; \gamma) & \text { for } & x^{0}<0,
\end{array}\right.
\end{array}
$$

d) for $\gamma$ real and larger than seventeen $E_{F(\bar{F}), r}(x ; \gamma)$ and $E_{r}^{( \pm)}(x ; \gamma)$ are locally $L^{4}$-integrable functions of $x$ such that products of the form

$$
\prod_{1 \leqq j<k \leqq 4}\left[1+i E_{r}^{\#}\left(x_{j}-x_{k} ; \gamma\right)\right]
$$

are unambiguously defined. Here, $E_{r}^{\sharp}\left(x_{j}-x_{k} ; \gamma\right)$ stands for either $E_{F, r}\left(x_{j}-x_{k} ; \gamma\right)$ or $-E_{\bar{F}, r}\left(x_{j}-x_{k} ; \gamma\right)$ or $E_{r}^{(+)}\left(x_{j}-x_{k} ; \gamma\right)$ or $-E_{r}^{(-)}\left(x_{j}-x_{k} ; \gamma\right)$.

In order to prove Eqs. (12) and (12') we start from the infinitely differentiable functions $E_{F(\bar{F}), r, \varepsilon}(x ; \gamma)$ which regularize the $L_{\text {loc }}^{4}$-regularizations $E_{F(\bar{F}), r}(x ; \gamma)$ even further: $E_{F(\bar{F}), r}(x ; \gamma)$ are the limits of $E_{F(\bar{F}), r, \varepsilon}(x ; \gamma)$ as $\varepsilon$ tends to +0 for $\gamma>17$ in the topology of $L_{\text {loc }}^{4}$. It follows from this fact that

$$
\left(\prod_{1 \leqq j<k \leqq 4}\left[1 \text { ( } i E_{F(\bar{F}), r}\left(x_{j}-x_{k} ; \gamma\right)\right]\right)_{\text {conn. }}
$$

are the limits of

$$
\left(\prod_{1 \leqq j<k \leqq 4}\left[1\left( \pm, i E_{F(\bar{F}), r, \varepsilon}\left(x_{j}-x_{k} ; \gamma\right)\right]\right)_{\mathrm{conn} .} \doteqdot \overline{(F)} i_{\widetilde{\widetilde{3}}_{r, \varepsilon}}\left(x_{1}, \ldots, x_{4} ; \gamma\right)\right.
$$

as $\varepsilon$ tends to +0 for $\gamma>17$ in the topology of $L_{\text {loc }}^{1}$.

We evaluate the Fourier transform $\widetilde{\widetilde{3}}_{r, \varepsilon}\left(p_{1}, \ldots, p_{4} ; \gamma\right)$ of $\widetilde{\overline{3}}_{r, \varepsilon}\left(x_{1}, \ldots, x_{4} ; \gamma\right)$ using standard techniques, i.e. we straighten out the contour $L_{\gamma}$ entering the definition of $E_{F(\bar{F}), r, \varepsilon}(x ; \gamma)$ (Eq. 53 of Ref. [2]), introduce Feynman parameters, work out the Gaussian integrals over the loop momenta, subdivide the integration region $\left(\otimes\left[0,+\infty[)^{b}\right.\right.$ of the Feynman parameters into sectors according to the respective ordering, set the $j^{\text {th }}$ smallest parameter equal to $\alpha_{j}=t_{j} \ldots t_{b-1} \cdot t_{b}$ with $t_{m} \in I m=1, \ldots, b-1$ and $0 \leqq t_{b}<+\infty$, absorb part of the orderings by permutations of the external momenta and finally perform the integration over $t_{b}$. Thus we 
establish that for $\gamma>17\left(\widetilde{\widetilde{3}}_{r, \varepsilon}\left(p_{1}, \ldots, p_{4} ; \gamma\right)\right.$ is equal to the r.h.s. of Eq. (9) with the only difference that for $\varrho \in \mathfrak{P}_{a 1}$

$$
\begin{aligned}
& {\left[-\frac{D_{a}^{\varrho}\left(\{\alpha\} ; p_{i}, p_{j}, p_{k}, p_{l}\right) / \alpha_{a} \ldots \alpha_{3}}{C_{a}^{\varrho}(\{\alpha\}) / \alpha_{a} \ldots \alpha_{4}} \overline{(\mp)} i 0\right]^{\sum^{a} s_{m}+2(a-3)} \text { is to be replaced by }} \\
& {\left[-\frac{D_{a}^{\varrho}\left(\{\alpha\} ; p_{i}, p_{j}, p_{k}, p_{l}\right) / \alpha_{a} \ldots \alpha_{3}}{C_{a}^{\varrho}(\{\alpha\}) / \alpha_{a} \ldots \alpha_{4}} \overline{\mp)} i \sum_{1}^{a} \varepsilon \frac{\alpha_{n}}{\alpha_{3}}\right]^{\sum_{1}^{\sum} s_{m}+2(a-3)},} \\
& \text { for } \varrho \in \mathfrak{P}_{a 2}\left[-\frac{D_{a}^{\varrho}\left(\{\alpha\} ; p_{i}, p_{j}, p_{k}, p_{l}\right) / \alpha_{a} \ldots \alpha_{3}}{C_{a}^{\varrho}(\{\alpha\}) / \alpha_{a} \ldots \hat{\alpha}_{4} \alpha_{3}} \bar{\mp} i 0\right]^{\frac{a}{\sum^{1} s_{m}+2(a-3)}} \text { by } \\
& {\left[-\frac{D_{a}^{\varrho}\left(\{\alpha\} ; p_{i}, p_{j}, p_{k}, p_{l}\right) / \alpha_{a} \ldots \alpha_{3}}{C_{a}^{\varrho}(\{\alpha\}) / \alpha_{a} \ldots \widehat{\alpha_{4} \alpha_{3}}} \overline{(-)} i \sum_{1}^{a} \varepsilon \frac{\alpha_{n}}{\alpha_{4}}\right]^{a}{ }^{\sum s_{m}+2(a-3)},} \\
& T_{0}^{\varrho( \pm)}\left(p_{i}, p_{k},-p_{i}-p_{k} ; s_{1}, s_{2}, s_{3}\right) \text { by } \int_{0}^{1} d t_{2} t_{2}^{s_{3}+1} \int_{0}^{1} d t_{1} t_{1}^{-s_{1}-1}\left[1+t_{2}+t_{2} t_{1}\right]^{-2} \\
& \cdot\left[-\frac{p_{\varrho(i)}^{2}+t_{1} p_{\varrho(k)}^{2}+t_{2} t_{1}\left(p_{\varrho(i)}+p_{\varrho(k)}\right)^{2}}{1+t_{2}+t_{2} t_{1}} \overline{(\mp)} i \varepsilon\left(t_{2}^{-1}+1+t_{1}\right)\right]^{\sum^{\sum s_{m}+2}} \text { and } \\
& {\left[- ( \Sigma ^ { \prime } p _ { m } ) ^ { 2 } ( \mp i 0 ] ^ { s _ { n } } \text { by } \left[-\left(\Sigma^{\prime} p_{m}\right)^{2}(\mp i \varepsilon]^{s_{n}}\right.\right. \text {. }}
\end{aligned}
$$

Here, the symbol $\Sigma^{\prime}$ stands for the respective partial sums.

$\widetilde{\sim}$ We observe that the $s_{n}$-integrations in the expression for $\overline{\overline{3}}_{r, \varepsilon}\left(p_{1}, \ldots, p_{n} ; \gamma\right)$ just established are uniformly convergent (in $\left.\varepsilon\right)$, that the powers which occur for any $t_{n}$ are larger than -1 and that

$$
-6<\sum_{1}^{a} \mathscr{R} e s_{n}+2(a-3)<0,-4<\sum_{1}^{3} \mathscr{R} e s_{n}+2<0,-2<\mathscr{R} e s_{n}<0
$$

$n=1, \ldots, a$. The lower bounds coincide with the restrictions on the applicability of the limit relations $(7) /(8),\left(7^{\prime}\right) /\left(8^{\prime}\right)$. The upper bounds guarantee that the integrands are (with respect to $\varepsilon$ ) uniformly bounded distribution-valued functions of $t_{n}$. In virtue of the theorem on bounded convergence, for $\gamma>17$ the following relation holds

$$
\lim _{\varepsilon \downarrow 0} \widetilde{\widetilde{3}}_{r, \varepsilon}\left(p_{1}, \ldots, p_{4} ; \gamma\right)=\widetilde{\widetilde{3}}_{r}\left(p_{1}, \ldots, p_{4} ; \gamma\right)
$$

or after Fourier transformation

$$
\lim _{\varepsilon \downarrow 0}\left(\overline{3}_{r, \varepsilon}\left(x_{1}, \ldots, x_{4} ; \gamma\right)=\overline{3}_{r}\left(x_{1}, \ldots, x_{4} ; \gamma\right) .\right.
$$


If we combine this result with the limit relation between the expression (13) and (14) we conclude for $\gamma>17$

$$
\overline{\mathfrak{Z}}_{r}\left(x_{1}, \ldots, x_{4} ; \gamma\right)={ }_{( \pm} i{ }_{1 \leqq j<k \leqq 4}\left[1\left( \pm, i E_{F(\bar{F}), r}\left(x_{j}-x_{k} ; \gamma\right)\right]\right)_{\text {conn. }}
$$

q.e.d.

Formal manipulations which are correct for $L_{\text {loc }}^{4}$-integrable functions yield for $\gamma>17$

a) the unitary relation

$$
\begin{aligned}
\frac{1}{2 i}\left\{\mathcal{Z}_{r}\left(x_{1}, \ldots, x_{4} ; \gamma\right)-\overline{\mathbf{Z}}_{r}\left(x_{1}, \ldots, x_{4} ; \gamma\right)\right\} \\
=-\frac{1}{2} \sum_{X}(-1)^{|X|}\left(\left\{\prod_{\substack{i<j \\
i, j \in X}}\left[1+i E_{F, r}\left(x_{i}-x_{j} ; \gamma\right)\right]\right\}\right. \\
\left.\cdot\left\{\prod_{\substack{k \in X \\
l \in Y}}\left[1+i E_{r}^{(+)}\left(x_{k}-x_{l} ; \gamma\right)\right]\right\}\left\{\prod_{\substack{m<n \\
m, n \in Y}}\left[1-i E_{\bar{F}, r}\left(x_{m}-x_{n} ; \gamma\right)\right]\right\}\right)_{\mathrm{conn}}
\end{aligned}
$$

where the sum runs over all partitions of the set $\{1,2,3,4\}$ into two disjoint non-empty subsets $X$ and $Y: X \cup Y=\{1,2,3,4\}, X \cap Y=\phi$.

b) The locality relations

i) $\begin{aligned} & 3_{r}\left(x_{1}, \ldots, x_{4} ; \gamma\right)=i\left(\left\{\prod_{\substack{i<j \\ i, j \in X}}\left[1+i E_{F, r}\left(x_{i}-x_{j} ; \gamma\right)\right]\right\}\right. \\ & \cdot\left\{\prod_{\substack{k \in X \\ l \in Y}}\left[1+i E_{r}^{(+)}\left(x_{k}-x_{l} ; \gamma\right)\right]\right\}\left\{\prod_{\substack{m<n \\ m, n \in Y}}\left[1+i E_{F, r}\left(x_{m}-x_{n} ; \gamma\right)\right]\right)_{c o n n .}\end{aligned}$

ii) $\overline{\mathbf{3}}_{r}\left(x_{1}, \ldots, x_{4} ; \gamma\right)=-i\left(\left\{\prod_{\substack{i<j \\ i, j \in X}}\left[1-i E_{\bar{F}}\left(x_{i}-x_{j} ; \gamma\right)\right]\right\}\right.$

$$
\left.\cdot\left\{\prod_{\substack{k \in X \\ l \in Y}}\left[1+i E_{r}^{(+)}\left(x_{l}-x_{k} ; \gamma\right)\right]\right\}\left\{\prod_{\substack{m<n \\ m, n \in Y}}\left[1-i E_{\bar{F}, r}\left(x_{m}-x_{n} ; \gamma\right)\right]\right\}\right)_{\text {conn. }}
$$

if $x_{i}^{0}>x_{m}^{0}$ for all $i \in X, m \in Y$. Here again, $(X, Y)$ denotes a partition of the set $\{1,2,3,4\}$ into two disjoint non-empty subsets.

As we already know, the left hand sides of these relations are ultra distribution-valued analytic functions of $\gamma$ in $W_{\delta, 4}$ and their limits exist in $\mathfrak{C}_{1 / 3}^{\prime}\left(\mathbb{R}^{16}\right)$ as $\gamma$ tends to +1 from $W_{\delta, 4}$. Also, the products on the right hand sides of these relations are ultra distribution-valued analytic functions of $\gamma$ in $W_{\delta, 4}$ and their limits exist in $\mathfrak{C}_{1 / 3}^{\prime}\left(\mathbb{R}^{16}\right)$ as $\gamma$ tends to +1 from $W_{\delta, 4}$.

In order to prove this, view the right hand sides of the unitary and locality relations as convolutions in momentum space, remember that the integrands, are analytic functions of $\gamma$ in $W_{\delta, 4}$, that their limits exist in $\mathfrak{M}_{1 / 3}^{\prime}\left(\mathbb{R}^{16}\right)$ as $\gamma$ tends to +1 from $W_{\delta, 4}$ and note that the integrations 
over the loop momenta are uniformly convergent when the right hand sides are tested with test functions from $\boldsymbol{C}_{1 / 3}\left(\mathbb{R}^{16}\right)$.

By the uniqueness of analytic continuation in simply connected regions we infer the appropriate unitary and locality relations for

$$
\overline{\mathfrak{Z}}_{r}\left(x_{1}, \ldots, x_{4}\right)=\lim _{\gamma \rightarrow 1, \gamma \in W_{\delta, 4}}\left(\overline{3}_{r}\left(x_{1}, \ldots, x_{4} ; \gamma\right)\right. \text {. }
$$

For the linear combination

$$
\begin{aligned}
(\overline{3})\left(x_{1}, \ldots, x_{4}\right)= & \frac{1}{128}\left(93 \sum_{r= \pm 1}-37 \sum_{r= \pm 3}+9 \sum_{r= \pm 5}-\sum_{r= \pm 7}\right) \\
& \cdot \lim _{\gamma \rightarrow 1, \gamma \in W_{\delta, 4}}\left(\overline{3}_{r}\left(x_{1}, \ldots, x_{4} ; \gamma\right)\right.
\end{aligned}
$$

we obtain the following relations in which $\tau\left(x_{1}, \ldots, x_{4}\right)$ stands for an arbitrary admissible definition of $i\left(\prod_{1 \leqq j<k \leqq 4}\left[1+i E_{F}\left(x_{j}-x_{k}\right)\right]\right)_{\text {conn. }}$.

a) unitarity:

$$
\begin{aligned}
& \frac{1}{2 i}\left\{3\left(x_{1}, \ldots, x_{4}\right)-\overline{3}\left(x_{1}, \ldots, x_{4}\right)\right\} \\
&=\frac{1}{2 i}\left\{\tau\left(x_{1}, \ldots, x_{4}\right)-\tau\left(x_{1}, \ldots, x_{4}\right)^{*}\right\} \\
& \quad+\frac{1}{2} \sum_{|X|=2}\left(\left\{i \lambda^{2} \pi^{3} g\left(-\frac{\lambda}{4} \square\right) \delta\left(x_{i}-x_{j}\right)\right\}_{i, j \in X}\right. \\
&\left.\cdot\left\{\prod_{\substack{k \in X \\
l \in Y}}\left[1+i E^{(+)}\left(x_{k}-x_{l}\right)\right]\right\}\left\{i \lambda^{2} \pi^{3} g\left(-\frac{\lambda}{4} \square\right) \delta\left(x_{m}-x_{n}\right)\right\}_{\substack{m<n \\
m, n \in Y}}\right)_{\text {conn. }}
\end{aligned}
$$

b) locality if $x_{i}^{0}>x_{m}^{0}$ for all $i \in X, m \in Y$

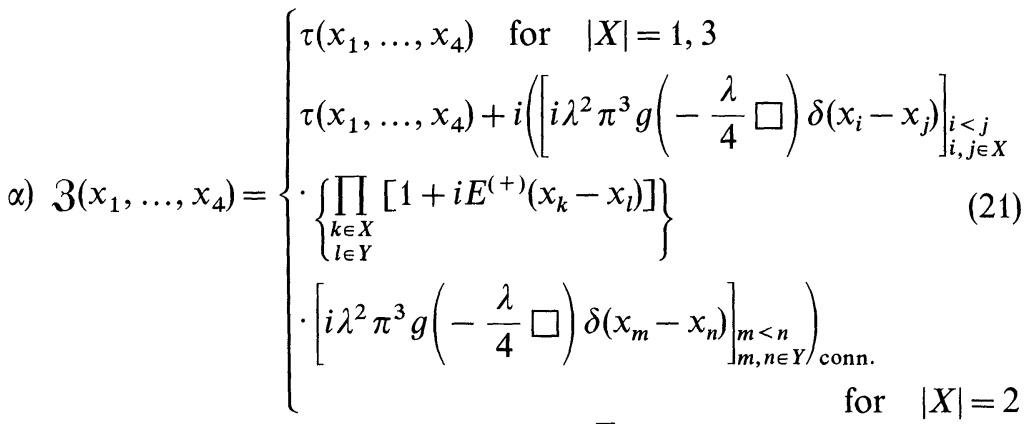

$\beta$ ) a corresponding locality relation for $\overline{3}\left(x_{1}, \ldots, x_{4}\right)$

c) reality: $\overline{3}\left(x_{1}, \ldots, x_{4}\right)=\left[3\left(x_{1}, \ldots, x_{4}\right)\right]^{*}$

d) symmetry: $3\left(x_{\sigma(1)}, \ldots, x_{\sigma(4)}\right)=\mathfrak{Z}\left(x_{1}, \ldots, x_{4}\right)$ for any $\sigma \in \mathfrak{S}_{4}$. 


\section{B. Asymptotics of the Real Part of $\tilde{\mathfrak{3}}\left(p_{1}, \ldots, p_{4}\right)$}

In this subsection we shall establish the asymptotic behavior of $\mathscr{R} e \tilde{\mathfrak{Z}}\left(p_{1}, \ldots, p_{4}\right)$ in the region where the Minkowski squares of all momenta together will all their partial sums are bounded below by some negative constant $-K^{2}$ and where at least one momentum or one partial sum of the momenta tends time-like to infinity. The methods developed in Ref. [2], Section III to study the analogous question for $\mathscr{R} e \tilde{\tau}\left(p_{1}, p_{2}, p_{3}\right)$ are good enough to control the asymptotics of

$$
\begin{aligned}
& \mathscr{R e} \tilde{\mathfrak{Z}}_{\underline{a}}\left(p_{1}, \ldots, p_{4}\right)=\frac{1}{128}\left(93 \sum_{r= \pm 1}-37 \sum_{r= \pm 3}+9 \sum_{r= \pm 5}-\sum_{r= \pm 7}\right) \\
& \lim _{\gamma \rightarrow 1, \gamma \in W_{\delta, 4}} \mathscr{R} e \tilde{\mathfrak{Z}}_{a, r}\left(p_{1}, \ldots, p_{4} ; \gamma\right)
\end{aligned}
$$

for $a=1,2,4,5,6$ in the above-mentioned region. For $a=3$, however, those methods do not suffice and a new technique has to be set up to clarify the asymptotic behavior of $\operatorname{Im} \tilde{\mathscr{T}}\left(p_{1}, p_{2}, p_{3}\right)$ in the region $\left\{\left(p_{1}, p_{2}, p_{3}\right) /\left(p_{m}\right)^{2}>-K^{2}, m=1,2,3\right\}$ the appearance of $\tilde{\mathscr{T}}\left(p_{1}, p_{2}, p_{3}\right)$ in the term $\widetilde{\mathcal{Z}}_{\underline{3}}$ being obvious.

By the methods of Ref. [2], Section III and II respectively, one can show that one commits only an error of type $0\left(\left(\operatorname{Max}\left\{p_{1}^{2}, p_{2}^{2}, p_{3}^{2}\right)^{-5 / 2}\right)\right.$ if one replaces $\mathscr{I}_{m} \tilde{\mathscr{T}}\left(p_{1}, \ldots, p_{3}\right)$ by $\mathscr{R} e \frac{i}{2}\left\{\tilde{\mathscr{T}}_{1}\left(p_{1}, p_{2}, p_{3} ; \gamma=1\right)\right.$ $\left.-\tilde{\mathscr{T}}_{-1}\left(p_{1}, p_{2}, p_{3} ; \gamma=1\right)\right\}$ and that this latter expression satisfies the following locality relation:

$$
\begin{aligned}
& \mathscr{R} e \frac{i}{2}\left\{\mathscr{T}_{1}\left(x_{1}, x_{2}, x_{3} ; \gamma=1\right)-\mathscr{T}_{-1}\left(x_{1}, x_{2}, x_{3} ; \gamma=1\right)\right\} \\
& =-\pi^{3} \lambda^{2} \mathscr{R} e i\left[i E^{(+)}\left(x_{i}-x_{j}\right)\right]\left[g\left(-\frac{\lambda}{4} \square\right) \delta\left(x_{j}-x_{k}\right)\right]\left[i E^{(+)}\left(x_{i}-x_{k}\right)\right]
\end{aligned}
$$

for $x_{i}^{0}$ larger than $x_{j}^{0}$ and $x_{k}^{0}$ and a similar locality relation for $x_{i}^{0}$ less than $x_{j}^{0}$ and $x_{k}^{0}$. Hence, outside the coincidence points $x_{1}=x_{2}=x_{3}$ the following relation is true

$$
\begin{aligned}
& \mathscr{R} e \frac{i}{2}\left\{\mathscr{T}_{1}\left(x_{1}, x_{2}, x_{3} ; \gamma=1\right)-\mathscr{T}_{-1}\left(x_{1}, x_{2}, x_{3} ; \gamma=1\right)\right\} \\
& =-\pi^{3} \lambda^{2} \sum_{\sigma \in \subseteq_{3}} \mathscr{R} e \frac{i}{2}\left[i E_{F}\left(x_{i}-x_{j}\right)\right]\left[g\left(-\frac{\lambda}{4} \square\right) \delta\left(x_{j}-x_{k}\right)\right]\left[i E_{F}\left(x_{k}-x_{i}\right)\right] .
\end{aligned}
$$

Next, we construct an extension of the r.h.s. (not necessarily agreeing with the 1.h.s.). As usual we do this via its Fourier transform. To this end, we represent the differential operator $g\left(-\frac{\lambda}{4} \square\right)$ as an integral over 
translation operators [9]:

$g\left(-\frac{\lambda}{4} \square_{\zeta}\right)=\frac{1}{2 \pi^{2} \lambda^{2}} \int_{\|\varrho\|^{2}=1} d \varrho \frac{1}{2 \pi i} \oint d \zeta \zeta^{3} e^{-\frac{\lambda}{\zeta^{2}}} e^{\zeta\left[\varrho^{0} \frac{\partial}{\partial \xi^{0}}+i \sum_{r=1}^{3} \varrho^{r} \frac{\partial}{\partial \xi^{r}}\right]}$,

employ standard techniques [8] to manipulate the Fourier transform of the r.h.s. and obtain for it the hitherto formal expression

$$
\begin{aligned}
& -\delta\left(\sum_{1}^{3} p_{m}\right) \sum_{\sigma \in \mathfrak{S}_{3}}\left\{2 \pi^{5 / 2} \sum_{v_{1}=0}^{\infty} \cdots \sum_{v_{3}=0}^{\infty}\left[\left(p_{j}-p_{k}\right)^{2}\right]^{v_{1}+v_{2}}\left[p_{i}^{2}\right]^{v_{2}}\left[p_{i} \cdot\left(p_{j}-p_{k}\right)\right]^{2 v_{3}}\right. \\
& \cdot c_{v_{1} \ldots v_{3}} \int d y y^{3+v_{1}+2 v_{2}+2 v_{3}} J_{1+2 v_{2}+2 v_{3}}\left(\sqrt{y} \sqrt{-p_{i}^{2}}\right)\left[\sqrt{y} \sqrt{-p_{i}^{2}}\right]^{-1-2 v_{2}-2 v_{3}} \\
& \cdot \frac{1}{2 \pi \mathrm{i}} \oint d \zeta \zeta^{1+v_{1}+v_{2}+v_{3}} \int_{0}^{\pi} d \Theta \sin ^{2} \Theta C_{2 v_{2}+2 v_{3}}^{1}(\cos \Theta) \\
& \left.\cdot \exp \left[-\frac{\lambda}{y} f(\zeta, \Theta)\right]+\pi^{3} \lambda^{2} g\left(\frac{\lambda}{4} p_{i}^{2}\right) \mathscr{R} e \tilde{E}_{F}\left(p_{j}\right)\right\} .
\end{aligned}
$$

Here we have set

$$
\begin{aligned}
c_{v_{1} \ldots v_{3}}= & (-1)^{v_{1}+v_{2}}\left(v_{2}+v_{3}\right) ! \\
& \cdot\left[v_{1} ! v_{2} ! v_{3} ! \Gamma\left(\frac{1}{2}+v_{3}\right)\left(1+v_{1}+2 v_{2}+2 v_{3}\right) ! \cdot 16^{v_{1}+v_{2}+v_{3}}\right]^{-1}, \\
f(\zeta, \Theta)= & \frac{1}{\zeta}+2\left(1+\frac{\zeta}{4}\right)\left[\left(1+\frac{\zeta}{4}\right)^{2}-\zeta \cos ^{2} \Theta\right]^{-1} .
\end{aligned}
$$

The symbol $J_{\mu}$ denotes the Bessel function of first kind and order $\mu$, while the symbol $C_{\mu}^{1}$ stands for the Gegenbauer polynomial. The integration runs along some path 0 to $+\infty$.

We may deform the $\zeta$-contour into the circle

$$
\left\{\zeta / \zeta=-\frac{2}{3}\left(1+e^{i \psi}\right),-\pi \leqq \psi \leqq+\pi\right\}
$$

without changing the value of the $\zeta$-integral. On this circle $\mathscr{R} e f$ is always non-negative. Inspite of the fact the $\mathscr{R} e f$ assumes the value 0 there (for $\Theta=0, \zeta=-4 / 3), \operatorname{aig} f \mid<\pi / 2$ is valid over the entire range of integration since the critical point is a saddle point and $f$ itself is equal to zero there.

With this information at hand, we may give a precise definition of the formal expression (25): the path of the $y$-integration is fixed to run from 0 to $+\infty$ along the positive axis.

From the integral representation (25) we can read off the asymptotic behavior of $\mathscr{R} e \frac{i}{2}\left\{\tilde{\mathscr{T}}_{1}-\tilde{\mathscr{T}}_{-1}\right\}$ in the region of interest which in turn settles the question of the asymptotic behavior of $\mathscr{I}_{m} \tilde{\mathscr{T}}\left(p_{1}, p_{2}, p_{3}\right)$, of $\mathscr{R} e \tilde{\mathfrak{Z}}_{\underline{3}}\left(p_{1}, \ldots, p_{4}\right)$ and finally of $\mathscr{R} e \tilde{\mathfrak{3}}\left(p_{1}, \ldots, p_{4}\right)$. We content ourselves with the statement of the asymptotic structure of $\mathscr{R} e \tilde{\mathfrak{Z}}$ in the region 
$\left\{\left(p_{1}, p_{2}, p_{3}, p_{4}\right) /\left(p_{i}\right)^{2}>-K^{2},\left(p_{j}+p_{k}\right)^{2}>-K^{2} i, j, k=1,2,3,4\right\}, K^{2}$ some positive constant

$$
\begin{aligned}
\mathscr{R} e & \tilde{\mathbf{Z}}\left(p_{1}, \ldots, p_{4}\right)=\delta\left(\sum_{1}^{4} p_{m}\right) \cdot O\left(\left(\operatorname{Max}_{i, j, k}\left\{p_{i}^{2},\left(p_{j}+p_{k}\right)^{2}\right\}\right)^{-c}\right) \\
+ & \delta\left(\sum_{1}^{4} p_{m}\right) \sum_{\sigma \in \mathfrak{S}_{4}} O\left(\left(p_{l}^{2}\right)^{-\infty}\right)\left\{\sum_{m=0}^{\infty} \sum_{n=0}^{\infty}\left[\left(p_{j}-p_{k}\right)^{2}\right]^{m}\left[\left(p_{j}-p_{k}\right) \cdot\left(p_{j}+p_{k}\right)\right]^{2 n}\right. \\
\cdot & \left.W_{m n}\left(\left(p_{j}+p_{k}\right)^{2}\right)\right]+\left[\begin{array}{l}
\left.\left(p_{j}-p_{k}\right) \rightarrow\left(p_{j}+2 p_{k}\right)\right] \\
\left(p_{j}+p_{k}\right) \rightarrow p_{j}
\end{array}\right] \\
+ & \left.\sum_{m_{1}=0}^{\infty} \sum_{m_{2}=0}^{\infty} \sum_{m_{3}=0}^{\infty} a_{m_{1} m_{2} m_{3}}\left(p_{j}^{2}\right)^{m_{1}}\left(p_{k}^{2}\right)^{m_{2}}\left(\left(p_{j}+p_{k}\right)^{2}\right)^{m_{3}}\right\} \\
+ & \delta\left(\sum_{1}^{4} p_{m}\right) \sum_{\sigma \in \mathfrak{S}_{4}}\left\{\sum_{m=0}^{\infty} \sum_{n_{1}=0}^{\infty} \ldots \sum_{n_{3}=0}^{\infty}\left(p_{i}^{2}\right)^{m}\left(p_{i} \cdot p_{l}\right)^{n_{1}}\left(p_{i} \cdot\left(p_{i}+p_{j}\right)\right)^{n_{2}}\right. \\
& \cdot\left(p_{l} \cdot\left(p_{i}+p_{j}\right)\right)^{n_{3}} W_{m n_{1} \ldots n_{3}}^{(1)}\left(p_{l}^{2},\left(p_{i}+p_{j}\right)^{2}, p_{k}^{2}\right)+a \text { similar term with } \\
& \text { the variables }\left(p_{i}, p_{l}, p_{i}+p_{j}, p_{k}\right) \text { replaced by }\left(p_{i}, p_{i}+p_{j}, p_{l}, p_{k}\right)+a \\
& \text { similar term with the variables }\left(p_{i}, p_{l}, p_{i}+p_{j}, p_{k}\right) \text { replaced } \\
& \text { by } \left.\left(p_{k}, p_{l}, p_{i}, p_{i}+p_{l}\right)\right\}
\end{aligned}
$$

where the "functions" $W_{m n}(y)$ and $W_{m n_{1} \ldots n_{3}}^{(r)}\left(y_{1}, y_{2}, y_{3}\right) r=1,2,3$ are of type $O\left(y^{-n-c}\right)$ and $O\left(y_{1}^{-\infty}, y_{2}^{-n_{2}-n_{3}-c}, y_{3}^{-d}\right)$ with $c \geqq 23 / 10, d \geqq 0$ and where $a_{m_{1} \ldots m_{3}}$ are real constants.

\section{The Deficiency Amplitudes: Construction and Asymptotics}

The occurrence of the terms

$$
\begin{gathered}
\left(\left[i \lambda^{2} \pi^{3} g\left(-\frac{\lambda}{4} \square\right) \delta\left(x_{i}-x_{j}\right)\right]_{\substack{i<j \\
i, j \in X}}\left\{\prod_{\substack{k \in X \\
l \in Y}}\left[1+i E^{(+)}\left(x_{k}-x_{l}\right)\right]\right\}\right. \\
\cdot\left[i \lambda^{2} \pi^{3} g\left(-\frac{\lambda}{4} \square\right) \delta\left(x_{m}-x_{n}\right)\right]_{\substack{m<n \\
m, n \in Y}} \text { conn. } \text { for }|X|=2
\end{gathered}
$$

in the unitarity and locality relations (20) and (21) indicates that the amplitudes $3\left(x_{1}, \ldots, x_{4}\right)$ and $\overline{3}\left(x_{1}, \ldots, x_{4}\right)$ do not provide admissible definitions for the vacuum expectation value of the chronological and antichronological product of four exponentials of the free scalar field respectively.

In this section we shall construct amplitudes $z\left(x_{1}, \ldots, x_{4}\right)$ and $\bar{z}\left(x_{1}, \ldots, x_{4}\right)$, called deficiency amplitudes, that account just for these 
extra terms, i.e. amplitudes with the following properties

o) $\left(\overline{3}\left(x_{1}, \ldots, x_{4}\right) \in \mathbb{C}^{\prime}\left(\mathbb{R}^{16}\right)\right.$, Lorentz invariant,

i) $\frac{1}{2 i}\left[\mathfrak{z}\left(x_{1}, \ldots, x_{4}\right)-\bar{z}\left(x_{1}, \ldots, x_{4}\right)\right]$

$$
\begin{aligned}
= & \frac{1}{2} \sum_{|X|=2}\left(\left[i \lambda^{2} \pi^{3} g\left(-\frac{\lambda}{4} \square\right) \delta\left(x_{i}-x_{j}\right)\right]_{i, j \in X}\right. \\
& \cdot\left\{\prod_{\substack{i<X \\
l \in Y}}\left[1+i E^{(+)}\left(x_{k}-x_{l}\right)\right]\right\} \\
& \left.\cdot\left[-i \lambda^{2} \pi^{3} g\left(-\frac{\lambda}{4} \square\right) \delta\left(x_{m}-x_{n}\right)\right]_{\substack{m<n \\
m, n \in Y}}\right)_{\text {conn. }}
\end{aligned}
$$

ii) if $x_{i}^{0}>x_{m}^{0}$ for all $i \in X, m \in Y$ :

a) $3\left(x_{1}, \ldots, x_{4}\right)=\left\{\begin{array}{l}0 \quad \text { for }|X|=1,|X|=3 \\ -i\left(\left[i \lambda^{2} \pi^{3} g\left(-\frac{\lambda}{4} \square\right) \delta\left(x_{i}-x_{j}\right)\right]_{\substack{i<j \\ i, j \in X}}\right. \\ \cdot\left\{\prod_{\substack{k \in X \\ l \in Y}}\left[1+i E^{(+)}\left(x_{k}-x_{l}\right)\right]\right\} \\ \left.\cdot\left[i \lambda^{2} \pi^{3} g\left(-\frac{\lambda}{4} \square\right) \delta\left(x_{m}-x_{n}\right)\right]_{\substack{m<n \\ m, n \in Y}}\right)_{\text {conn. }} \text { for }|X|=2 .\end{array}\right.$

$\beta$ ) a corresponding relation for $\bar{z}\left(x_{1}, \ldots, x_{4}\right)$,

iii) $\bar{\jmath}\left(x_{1}, \ldots, x_{4}\right)=\left[\mathfrak{z}\left(x_{1}, \ldots, x_{4}\right)\right]^{*}$,

iv) $\mathfrak{z}\left(x_{\sigma(1)}, \ldots, x_{\sigma(4)}\right)=\mathfrak{z}\left(x_{1}, \ldots, x_{4}\right)$ for any $\sigma \in \mathfrak{S}_{4}$.

By these requirements $(-))\left(x_{1}, \ldots, x_{4}\right)$ is only determined up to an arbitrary real, Lorentz invariant, symmetric ultra distribution with support in the points $x_{1}=x_{2}=x_{3}=x_{4}$ and contained in the class $\mathfrak{C}_{1 / 3}^{\prime}\left(\mathbb{R}^{16}\right)$. We shall show that among the ultra distributions with properties o), ...,iv) there exists a least singular one: $\left(\overline{3}_{0}\right)\left(x_{1}, \ldots, x_{4}\right)$. Moreover, the definition of $(\overrightarrow{3}) 0\left(x_{1}, \ldots, x_{4}\right)$ is unique.

The locality relation ii) requires $\mathfrak{z}\left(x_{1}, \ldots, x_{4}\right)$ and $\overline{\mathfrak{z}}\left(x_{1}, \ldots, x_{4}\right)$ to be equal to

$$
\begin{gathered}
( \pm) i \frac{\lambda^{4} \pi^{6}}{8} \sum_{\sigma \in \mathfrak{S}_{4}}\left[g\left(-\frac{\lambda}{4} \square\right) \delta\left(x_{i}-x_{j}\right)\right]\left[g\left(-\frac{\lambda}{4} \square\right) \delta\left(x_{k}-x_{l}\right)\right] \\
\left.\cdot\left(\left\{\prod_{m=i, j} \prod_{n=k, l}\left[1( \pm)_{(}\right) E_{F(\bar{F})}\left(x_{m}-x_{n}\right)\right]\right\}-1\right)
\end{gathered}
$$


away from $x_{1}=x_{2}=x_{3}=x_{4}$. There, outside the coincidence points, the so defined quantities $3\left(x_{1}, \ldots, x_{4}\right)$ and $\bar{z}\left(x_{1}, \ldots, x_{4}\right)$ match all the requirements o), ...,iv).

Next, we have to extend the definitions of $3\left(x_{1}, \ldots, x_{4}\right)$ and $\bar{z}\left(x_{1}, \ldots, x_{4}\right)$ to Lorentz invariant ultra distributions over the entire $\mathbb{R}^{16}$ preserving the relations iii) and iv) and ensuring that the extensions are contained in the class $\mathbb{C}_{1 / 3}^{\prime}\left(\mathbb{R}^{16}\right)$. For any such extension, the unitarity relation is satisfied if and only if its Fourier transform is real whenever the external momenta are totally space-like.

We give the particular extensions $z_{0}$ and $\bar{\jmath}_{0}$ in terms of their Fourier transforms. For that, as before, we represent the differential operator $g\left(-\frac{\lambda}{4} \square\right)$ as an integral over translation operators [9]:

$g\left(-\frac{\lambda}{4} \square_{\xi}\right)=\frac{1}{2 \pi^{2} \lambda^{2}} \int_{\|\varrho\|^{2}=1} d \varrho \frac{1}{2 \pi i} \oint d \zeta \zeta^{3} e^{-\frac{\lambda}{\zeta^{2}} e^{\zeta}\left[\varrho^{0} \frac{\partial}{\partial \xi^{0}}+i \sum_{r=1}^{3} \varrho^{r} \frac{\partial}{\partial \xi^{r}}\right]}$,

apply the Gelfand Shilov procedure [8] and arrive after standard manipulations at the following expression for

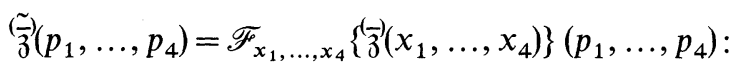

$$
\begin{aligned}
& (\tilde{\overline{3}})\left(p_{1}, \ldots, p_{4}\right)=\delta\left(\sum_{1}^{4} p_{m}\right) \sum_{\sigma \in \mathfrak{S}_{4}} \int d y z_{\sigma}\left(y ; p_{1}, \ldots, p_{4}\right) \\
& \text { (F) } i \frac{\lambda^{4} \pi^{6}}{8} \sum_{\sigma \in \mathscr{G}_{4}} \delta\left(p_{i}+p_{j}\right) g\left(\frac{\lambda}{4} p_{i}^{2}\right) \cdot \delta\left(p_{k}+p_{l}\right) g\left(\frac{\lambda}{4} p_{k}^{2}\right)
\end{aligned}
$$

where the integral should be taken along some path running from 0 to $\operatorname{sgn}\left(-\left(p_{i}+p_{j}\right)^{2}\right) \cdot \infty$ and where $z_{\sigma}\left(y ; p_{1}, \ldots, p_{4}\right)$ stands for

$$
\begin{gathered}
\frac{y^{5} \pi^{2}}{4^{3}} \int_{0}^{\pi / 2} d \Theta_{1} \sin ^{2} \Theta_{1} \int_{0}^{\pi / 2} d \Theta_{2} \sin ^{2} \Theta_{2} \frac{1}{2 \pi i} \oint d \zeta_{1} \zeta_{1} \frac{1}{2 \pi i} \oint d \zeta_{2} \zeta_{2} \int_{-1}^{+1} d \xi \\
\exp \left(\frac{\lambda}{4} f\left(\zeta_{1}, \zeta_{2}, \Theta_{1}, \Theta_{2}, \xi\right)\right) J_{\sigma}\left(y ; \zeta_{1}, \zeta_{2}, \Theta_{1}, \Theta_{2}, \xi ; p_{1}, \ldots, p_{4}\right)
\end{gathered}
$$

with

$$
\begin{aligned}
& f\left(\zeta_{1}, \zeta_{2}, \Theta_{1}, \Theta_{2}, \xi\right)=\frac{1}{\zeta_{1}}+\frac{1}{\zeta_{2}}+\sum_{x=1}^{2} \sum_{\lambda=1}^{2}\left[1+(-1)^{x} \sqrt{\zeta_{1}} \cos \Theta_{1}\right. \\
& +(-1)^{\lambda} \sqrt{\zeta_{2}} \cos \Theta_{2}+\frac{\zeta_{1}}{4} \\
& \left.+\frac{1}{2}(-1)^{x+\lambda} \sqrt{\zeta_{1} \zeta_{2}}\left(\cos \Theta_{1} \cos \Theta_{2}+\sin \Theta_{1} \sin \Theta_{2} \cdot \xi\right)+\frac{\zeta_{2}}{4}\right]^{-1}
\end{aligned}
$$


and

$$
\begin{aligned}
& J_{\sigma}\left(y ; \zeta_{1}, \zeta_{2}, \Theta_{1}, \Theta_{2}, \xi ; p_{1}, \ldots, p_{4}\right)=\sum_{v_{1}=0}^{\infty} \ldots \sum_{v_{8}=0}^{\infty} c_{v_{1}, \ldots, v_{8}} \\
& \cdot h_{v_{1}, \ldots, v_{8}}^{\sigma}\left(y ; \zeta_{1}, \zeta_{2}, \Theta_{1}, \Theta_{2}, \xi ; p_{1}, \ldots, p_{4}\right) J_{1+2 v_{6}+2 v_{7}}\left(\sqrt{y} \sqrt{-\left(p_{i}+p_{j}\right)^{2}}\right) \\
& \cdot\left[\sqrt{y} \sqrt{-\left(p_{i}+p_{j}\right)^{2}}\right]^{-1-2 v_{7}} .
\end{aligned}
$$

The symbol $J_{n}(x)$ stands for the Bessel function of first kind and $n^{\text {th }}$ order. The definition of $c_{v_{1}, \ldots, v_{8}}$ and $h_{v_{1}, \ldots, v_{8}}^{\sigma}\left(y ; \zeta_{1}, \zeta_{2}, \Theta_{1}, \Theta_{2}, \xi ; p_{1}, \ldots, p_{4}\right)$ can be found in Ref. [10]: Eqs. (34)-(36). $h_{v_{1}, \ldots, v_{8}}^{\sigma}$ is a polynomial in the variable $y$ and in the scalar products of the momenta.

$$
\begin{aligned}
& {\left[c_{v_{1}, \ldots, v_{8}} h_{v_{1}, \ldots, v_{8}}^{\sigma}\left(y ; \zeta_{1}, \zeta_{2}, \Theta_{1}, \Theta_{2}, \xi ; p_{1}, \ldots, p_{4}\right)\right]^{*} } \\
= & c_{v_{1}, \ldots, v_{8}} h_{v_{1}, \ldots, v_{8}}^{\sigma}\left(y^{*} ; \zeta_{1}^{*}, \zeta_{2}^{*}, \Theta_{1}^{*}, \Theta_{2}^{*}, \xi^{*} ; p_{1}^{*}, \ldots, p_{4}^{*}\right) .
\end{aligned}
$$

Furthermore, $c_{v_{1}, \ldots, v_{8}}$ and $h_{v_{1}, \ldots, v_{8}}^{\sigma}$ are such that a definition of $\left(\tilde{\tilde{z}}\left(p_{1}, \ldots, p_{4}\right)\right.$ contained in the class $\mathfrak{M}_{1 / 3}^{\prime}\left(\mathbb{R}^{16}\right)$ is possible.

Now, the extension problem poses itself in the following form: Give a precise definition of the integral on the r.h.s. of Eq. (30).

In order to do so we need some information about the behavior of $z_{\sigma}\left(y ; p_{1}, \ldots, p_{4}\right)$ in the neighborhood of $y=0$. We observe that for $y$ real and negative the $\zeta_{n}$-contours of integration in expression (31) may be deformed into contours $C_{\Theta_{1}, \Theta_{2}}^{n}$ given by

$C_{\Theta_{1}, \Theta_{2}}^{n}=\left\{\zeta_{n} / \zeta_{n}=-\varrho_{n}^{2}\left(1+e^{i \psi_{n}}\right),-\pi \leqq \psi_{n} \leqq+\pi, \varrho_{n}^{2}=\varrho_{n}^{2}\left(\Theta_{1}, \Theta_{2}\right)>0\right\}$

$n=1,2$ without changing the value of $z_{\sigma}\left(y ; p_{1}, \ldots, p_{4}\right)$. In particular, setting $\left(\varrho_{1}\right)^{2}=\frac{2}{3},\left(\varrho_{2}\right)^{2}=\frac{2}{3}$ in the vicinity of $\Theta_{1}=\Theta_{2}=0$,

$$
\begin{aligned}
& \varrho_{1}^{2}=\frac{1}{2}\left[1+\frac{\sin \left(\Theta_{1}-\Theta_{2}\right)}{\sqrt{4-\cos ^{2}\left(\Theta_{1}-\Theta_{2}\right)}}\right] \\
& \varrho_{2}^{2}=\frac{1}{2}\left[1-\frac{\sin \left(\Theta_{1}-\Theta_{2}\right)}{\sqrt{4-\cos ^{2}\left(\Theta_{1}-\Theta_{2}\right)}}\right]
\end{aligned}
$$

otherwise, $\mathscr{R e} f\left(\zeta_{1}, \zeta_{2}, \Theta_{1}, \Theta_{2}, \xi\right)$ is non-negative for the entire range of integration and $\left|\operatorname{aig} f\left(\zeta_{1}, \zeta_{2}, \Theta_{1}, \Theta_{2}, \xi\right)\right|<\frac{\pi}{2}$. [There is a one-dimensional continuum of saddle points with $f=0: \xi=+1, \operatorname{tg} \Theta_{1} \cdot \operatorname{tg} \Theta_{2}=3$, $\left(\zeta_{1}, \zeta_{2}\right)=\left(-2\left(\varrho_{1}\right)^{2},-2\left(\varrho_{2}\right)^{2}\right)$. Apart from these angles $\Theta_{1}$ and $\Theta_{2}, \mathscr{R e} f$ assumes the value 0 again only for $\Theta_{1}=0=\Theta_{2}: \zeta_{1}=-\frac{4}{3}$, $\zeta_{2}=\frac{2}{3} \lim _{\psi \rightarrow \pm \pi}\left(1+e^{i \psi}\right) \quad$ and $\quad \zeta_{1}=-\frac{2}{3} \lim _{\psi \rightarrow \pm \pi}\left(1+e^{i \psi}\right), \quad \zeta_{2}=-\frac{4}{3} \quad$ leaving $\mid$ aig $f \mid<\frac{\pi}{2}$, however.] Hence, $z_{\sigma}\left(y ; p_{1}, \ldots, p_{4}\right)$ stays finite as we approach the point $y=0$ along the negative axis. 
Now, we are able to give the precise interpretation of the integral on the r.h.s. of Eq. (30):

$$
\frac{1}{2}\left(\int_{e^{i \pi \cdot 0}}^{\operatorname{sgn}\left(-\left(p_{i}+p_{j}\right)^{2}\right) \cdot \infty}+\int_{e^{-i \pi \cdot 0}}^{\infty}\right) d y z_{\sigma}\left(y ; p_{1}, \ldots, p_{4}\right) .
$$

Obviously, this particular extension $\left(\frac{\tilde{\tilde{z}}}{\partial_{0}}\left(p_{1}, \ldots, p_{4}\right)\right.$ satisfies the requirements o), ii), iii), and iv). Moreover, in view of the reality of $(\tilde{\tilde{z}})\left(p_{1}, \ldots, p_{4}\right)$ for totally space-like momenta, the unitarity relation i) also holds true, i.e. $\tilde{z}_{0}$ and $\tilde{\bar{z}}_{0}$ just introduced are admissible definitions. $\tilde{z}_{0}$ and $\tilde{\bar{z}}_{0}$ are distinguished from all other possible definitions by the fact that $\mathscr{R} e^{(\tilde{\tilde{z}})}$ has the special structure

$$
\begin{aligned}
\mathscr{R}_{e}^{((\tilde{)})}\left(p_{1}, \ldots, p_{4}\right)=\delta\left(\sum_{1}^{4} p_{m}\right)\left\{\sum_{m_{2}=0}^{\infty} \ldots \sum_{n_{3}=0}^{\infty} w_{m_{2} \ldots n_{3}}\left(\left(p_{1}+p_{2}\right)^{2}\right)\right. \\
\cdot\left[\left(p_{2}+p_{3}\right)^{2}\right]^{m_{2}}\left[\left(p_{3}+p_{1}\right)^{2}\right]^{m_{3}}\left[\left(p_{1}+p_{2}\right) \cdot\left(p_{2}+p_{3}\right)\right]^{n_{1}} \\
\cdot\left[\left(p_{2}+p_{3}\right) \cdot\left(p_{3}+p_{1}\right)\right]^{n_{2}}\left[\left(p_{3}+p_{1}\right) \cdot\left(p_{1}+p_{2}\right)\right]^{n_{3}} \\
+ \text { cyclic permutations of }\{1,2,3\}\}
\end{aligned}
$$

where the sum runs over those indices only for which $n_{1}+n_{2}$ and $n_{2}+n_{3}$ (and $\left.n_{3}+n_{1}\right)$ are even and where for all such indices $w_{m_{2} \ldots n_{3}}\left(q^{2}\right)$ are realvalued "functions" of type $0\left(\left(q^{2}\right)^{-m_{2} \ldots-n_{3}-7}\right)$ for large time-like $q$, with the property $w_{m_{2} m_{3} n_{1} n_{2} n_{3}}=w_{m_{3} m_{2} n_{3} n_{2} n_{1}}$.

Any other possible definition of $\left(\frac{\tilde{\bar{z}}}{3}\left(p_{1}, \ldots, p_{4}\right)\right.$ differs from $(\tilde{\tilde{z}})\left(p_{1}, \ldots, p_{4}\right)$ by a real entire function

$$
\begin{aligned}
& \delta\left(\sum_{1}^{4} p_{m}\right) \sum_{m_{1}=0}^{\infty} \cdots \sum_{n_{3}=0}^{\infty} A_{m_{1} \ldots n_{3}}\left[\left(p_{1}+p_{2}\right)^{2}\right]^{m_{1}}\left[\left(p_{2}+p_{3}\right)^{2}\right]^{m_{2}}\left[\left(p_{3}+p_{1}\right)^{2}\right]^{m_{3}} \\
& \cdot\left[\left(p_{1}+p_{2}\right) \cdot\left(p_{2}+p_{3}\right)\right]^{n_{1}}\left[\left(p_{2}+p_{3}\right) \cdot\left(p_{3}+p_{1}\right)\right]^{n_{2}}\left[\left(p_{3}+p_{1}\right) \cdot\left(p_{1}+p_{2}\right)\right]^{n_{3}}
\end{aligned}
$$

where the summation is restricted by the same conditions as before and where for all summation indices satisfying these conditions

$$
A_{m_{1} m_{2} m_{3} n_{1} n_{2} n_{3}}=A_{m_{2} m_{3} m_{1} n_{2} n_{3} n_{1}}=A_{m_{1} m_{3} m_{2} n_{3} n_{2} n_{1}} .
$$

\section{Definition of the Time-Ordered Vacuum Expectation Value of Four Exponentials}

By construction, the sum of the auxiliary amplitude $(\overline{3})$ and an arbitrary deficiency amplitude $\left(\frac{-)}{\mathfrak{z}}\right.$ yields an admissible definition of the connected part of the vacuum expectation value of the chronological, respectively 
the antichronological product of four exponentials, i.e. $\left(\overline{3}\left(x_{1}, \ldots, x_{4}\right)\right.$ $+(-))\left(x_{1}, \ldots, x_{4}\right)$ satisfies

O) $\stackrel{(}{\mathfrak{3}}\left(x_{1}, \ldots, x_{4}\right)+(\overrightarrow{3})\left(x_{1}, \ldots, x_{4}\right) \in \mathfrak{C}_{1 / 3}^{\prime}\left(\mathbb{R}^{16}\right)$, Lorentz invariant

I) unitarity

II) locality

III) reality: $\left[3\left(x_{1}, \ldots, x_{4}\right)+z\left(x_{1}, \ldots, x_{4}\right)\right]^{*}=\overline{3}\left(x_{1}, \ldots, x_{4}\right)+\bar{z}\left(x_{1}, \ldots, x_{4}\right)$

IV) symmetry with respect to permutations of the arguments.

Conversely, every admissible definition can be obtained in this way.

Now, let us average the real part of an arbitrary given admissible definition with a real analytic test function $f \in Z\left(\mathbb{R}^{12}\right)$ in the spatial difference variables. From the asymptotic behavior of $\mathscr{R} e \tilde{\mathfrak{Z}}\left(p_{1}, \ldots, p_{4}\right)$ [Eq. (29)] and of $\mathscr{R} e \tilde{\mathfrak{z}}\left(p_{1}, \ldots, p_{4}\right)$ [Eqs. (39) and (40)] in the region $\left\{\left(p_{1}, \ldots, p_{4}\right) /\left(p_{i}\right)^{2}>-K^{2},\left(p_{j}+p_{k}\right)^{2}>-K^{2}, i, j, k=1, \ldots, 4\right\}$ where $K^{2}$ is some positive constant, we conclude (the various series appearing in those equations converge sufficiently uniformly) that the resulting distribution in the time-difference variables has the following structure: It consists of

a background, once continuously differentiable throughout $\mathbb{R}^{3}$ $+\delta$-derivative type singularities concentrated on planes $x_{i}^{0}=x_{j}^{0}$ (two coinciding times) each one multiplied by a three times continuously differentiable function of the coordinates of the respective plane $+\delta$-derivative type singularities concentrated on the lines $x_{i}^{0}=x_{j}^{0}=x_{k}^{0}$ (three coinciding times) or $x_{i}^{0}=x_{j}^{0}, x_{k}^{0}=x_{l}^{0}$ (two pairs of coinciding times) each one multiplied by a three times continuously differentiable function of the coordinate describing the movement along the line

$+\delta$-derivative type singularities attached to the point $x_{1}^{0}=x_{2}^{0}=x_{3}^{0}=x_{4}^{0}$.

To visualize the position of the singularities in the three dimensional space of the time differences, the variables

$$
\begin{aligned}
\xi_{1}=\frac{x_{1}^{0}+x_{2}^{0}}{2}-\frac{x_{3}^{0}+x_{4}^{0}}{2}, \quad \xi_{2} & =\frac{x_{1}^{0}+x_{3}^{0}}{2}-\frac{x_{2}^{0}+x_{4}^{0}}{2}, \\
\xi_{3} & =\frac{x_{1}^{0}+x_{4}^{0}}{2}-\frac{x_{2}^{0}+x_{3}^{0}}{2}
\end{aligned}
$$

are suited best for a symmetric plot.

The structure mentioned above expresses just the fact that all singularities of the spatially averaged real part can be separated from each other and from the background. Moreover, this separability condition does not only hold for real analytic spatial test functions $f \in Z\left(\mathbb{R}^{12}\right)$, but also holds for every real spatial test function $f \in \mathbb{C}_{1 / 3}\left(\mathbb{R}^{12}\right)$.

Now, among all possible choices for the connected part of the timeordered vacuum expectation value of four exponentials there is a particular one: $3+z_{0}$ which is distinguished from the rest by the fact that when its real part is averaged in the spatial difference variables with an 
arbitrary real test function $f \in \mathfrak{C}_{1 / 3}\left(\mathbb{R}^{12}\right)$ this average never contains $\delta$-derivative type singularities solely attached to the times $x_{1}^{0}=x_{2}^{0}$ $=x_{3}^{0}=x_{4}^{0}$. Thus the definition

$$
\tau\left(x_{1}, \ldots, x_{4}\right)=3\left(x_{1}, \ldots, x_{4}\right)+3_{0}\left(x_{1}, \ldots, x_{4}\right)
$$

is singled out in a unique way by the criterion of minimal singularity. On the basis of arguments given in Ref. [1] we expect this definition to lead to the simplest dynamics associated with the classical exponential Lagrangian.

We would like to conclude with two remarks: First, as a corollary of the discussion of Section IIIB we obtain the assertion that also the space averaged imaginary part of $\tau\left(x_{1}, x_{2}, x_{3}\right)$ enjoys the separability property (as well as the space averaged imaginary part of $\tau\left(x_{1}, x_{2}\right)$ ). However, we are not free to use this separability for a minimal definition of $\operatorname{Im} \tau$. Second, since the $s$-integrations in the equations defining $\tilde{\mathcal{Z}}_{a, r} a=4,5,6$ when bent around the real axis from -1 to $+\infty$ are uniformly convergent as the momenta vary inside compact sets, the analyticity structure of the (crossed and uncrossed) box graph amplitudes in the invariant momenta on all sheets of the Riemann surface is just the same as renormalizable models.

Acknowledgements. The author is greatly indebted to Dr. R. Flume for suggesting the potential usefulness of Efimov's representation of functions of d'Alembertians by means of translation operators and to Dr. D. Lüke and Mr. M. Schlindwein for numerically supporting the search for contours (37), (38).

It is a pleasure to thank the members of the Physics Department of the Federal University of Rio Grande do Sul, Brazil for their kind hospitality and Banco National do Desenvolvimento Econômico, Brazil for financial support.

\title{
References
}

1. Lehmann, H., Pohlmeyer, K.: Commun. math. Phys. 20, 101 (1971)

2. Pohlmeyer, K.: Commun. math. Phys. 26, 130 (1972)

3. Daniel,M., Mitter,P.K.: Math. Phys. 13, 1026 (1972)

4. Volkov, M. K.: JINR preprint E-2-6728 Dubna (1972)

5. Bogoliubov, N.N., Shirkov,D.V.: Introduction to the theory of quantized fields: New York: Interscience Publishers, Ltd. 1959

6. Jaffe, A.: Phys. Rev. 158, 1454 (1967) and unpublished manuscript

7. Speer,E. R.: J. Math. Phys. 9, 1404 (1968)

8. Gelfand,I.M., Schilow, G.E.: Verallgemeinerte Funktionen (Distributionen). Berlin: VEB Deutscher Verlag der Wissenschaften 1960

9. Efimov, G.V.: Commun. math. Phys. 7, 138 (1968)

10. Pohlmeyer, K.: DESY Report $73 / 53$

Communicated by K. Symanzik

\author{
K. Pohlmeyer \\ II. Institut für Theoretische Physik \\ D-2000 Hamburg 50 \\ Luruper Chaussee 149 \\ Federal Republic of Germany
}

